\title{
Sound scattering by several zooplankton groups. II. Scattering models
}

\author{
Timothy K. Stanton and Dezhang Chu \\ Department of Applied Ocean Physics and Engineering, Woods Hole Oceanographic Institution, \\ Woods Hole, Massachusetts 02543-1053 \\ Peter H. Wiebe \\ Department of Biology, Woods Hole Oceanographic Institution, Woods Hole, Massachusetts 02543-1049
}

(Received 22 March 1996; accepted for publication 3 November 1996)

\begin{abstract}
Mathematical scattering models are derived and compared with data from zooplankton from several gross anatomical groups-fluidlike, elastic shelled, and gas bearing. The models are based upon the acoustically inferred boundary conditions determined from laboratory backscattering data presented in part I of this series [Stanton et al., J. Acoust. Soc. Am. 103, 225-235 (1998)]. The models use a combination of ray theory, modal-series solution, and distorted wave Born approximation (DWBA). The formulations, which are inherently approximate, are designed to include only the dominant scattering mechanisms as determined from the experiments. The models for the fluidlike animals (euphausiids in this case) ranged from the simplest case involving two rays, which could qualitatively describe the structure of target strength versus frequency for single pings, to the most complex case involving a rough inhomogeneous asymmetrically tapered bent cylinder using the DWBA-based formulation which could predict echo levels over all angles of incidence (including the difficult region of end-on incidence). The model for the elastic shelled body (gastropods in this case) involved development of an analytical model which takes into account irregularities and discontinuities of the shell. The model for gas-bearing animals (siphonophores) is a hybrid model which is composed of the summation of the exact solution to the gas sphere and the approximate DWBA-based formulation for arbitrarily shaped fluidlike bodies. There is also a simplified ray-based model for the siphonophore. The models are applied to data involving single pings, ping-to-ping variability, and echoes averaged over many pings. There is reasonable qualitative agreement between the predictions and single ping data, and reasonable quantitative agreement between the predictions and variability and averages of echo data. (C) 1998 Acoustical Society of
\end{abstract} America. [S0001-4966(97)01110-7]

PACS numbers: 43.30.Ft, 43.30.Sf, 43.20.Fn [JHM]

\section{LIST OF SYMBOLS}

$a$

$\bar{a}$

$\alpha_{B}, T_{B}, C_{B}$

radius of sphere or cylinder

average radius

numerically determined coefficients in raybased bent fluid cylinder model

$\alpha_{L} \quad$ real part of $\nu_{L}$

$b_{j}$

$b_{m}^{(f)}$

$\beta$

$\beta_{\text {tilt }}$

$\beta_{L}$ scattering amplitude of local facet that is broadside to incident beam

modal series coefficient for homogeneous fluid sphere

L/a

tilt angle of infinitesimally thin disk or cross section of body at an arbitrary point $\mathbf{r}_{\text {pos }}$. This is the angle between the direction of the incident wave $\hat{k}_{i}$ and the plane containing the disk. Specifically, $\beta_{\text {tilt }}=90^{\circ}-\cos ^{-1}\left(\hat{k}_{i} \cdot \hat{r}_{\text {tan }}\right)$ where $\hat{r}_{\text {tan }}$ is the tangent to the body axis at point $\mathbf{r}_{\text {pos }}$ ( $\beta_{\text {tilt }}=0$ corresponds to broadside incidence to the disk axis at the arbitrary point on the body axis). $\beta_{\text {tilt }}$ is not to be confused with the orientation angle, $\theta$, of the body, although the two are the same when the body axis is straight. imaginary part of $\nu_{L}$; attenuation coefficient of
Lamb wave on elastic shelled sphere

$\beta_{L}^{\prime}(\infty) \quad$ attenuation coefficient of Lamb wave on flat plate $(a=\infty)$

sound speed

sound speed of Lamb wave

center-to-center distance between bubbles

deviation in effective radius from mean radius of rough sphere

phase shift due to partial circumnavigation (i.e., path between $\pm \theta_{L}$ points) of Lamb waves distance between the point of scatter and the zero phase reference plane $\left(\epsilon_{j}\right.$ is negative for points on the source/receiver side of zero phase reference plane)

$f \quad$ scattering amplitude

$f_{\text {bs }} \quad$ scattering amplitude in backscattering direction

$F_{L} \quad$ factor, ranging in value from 0 to 1 , to account for loss of Lamb wave due to discontinuity in shell

$g \quad \rho_{2} / \rho_{1}$

$\gamma_{\kappa}, \gamma_{\rho} \quad$ material property parameters in DWBA formulation

$G_{L} \quad$ coupling coefficient for combination of landing and launching of Lamb waves on shell 
$\mathbf{k}_{i}$

$\mathbf{k}_{s}$

$\underline{L}$

$\bar{L}$

$\lambda$

$\mu$

$\nu_{L}$

$\Phi_{L}$

$\phi_{M}$

$\rho_{c}$

$\rho$

$\mathbf{r}_{\text {pos }}$

$\mathbf{r}_{v}$

$\mathscr{B}_{12}$ $c_{2} / c_{1}$

$\sqrt{-1}$ unless used as a summation index or subscript to $\mathbf{k}$

acoustic wave number $(=2 \pi / \lambda)$

compressibility

wave number vector of incident field

wave number vector of scattered field

length of body

average length of body

acoustic wavelength

semi-empirical phase shift term for ray model

complex root of denominator of modal series coefficient for fluid-filled elastic spherical shell

phase shift of Lamb wave heuristically added

for nonideal body

meridional angle

radius of curvature of longitudinal axis of uniformly bent cylinder

mass density

position vector of axis of deformed cylinder

position vector of volume

plane wave/plane interface reflection coefficient (reflection off of medium ' 2 " due to in-

$s_{\theta}, s_{L}$
$s$
$\sigma$
$\left(\sigma_{b s}\right)_{1}$
$\left(\sigma_{b s}\right)_{N}$
$\mathrm{TS}$
$T_{12}, T_{21}$

$\theta$
$\theta_{L}$
$v$
$\zeta$
1,2
$\langle\cdots\rangle$

cident beam in medium " 1 ") $\left[=\left(\rho_{2} c_{2} / \rho_{1} c_{1}\right.\right.$ $\left.-1) /\left(\rho_{2} c_{2} / \rho_{1} c_{1}+1\right)\right]$

standard deviation of angle of orientation and length, respectively

$s_{L} / \bar{L}$

$\left\langle\Delta_{a}^{2}\right\rangle^{1 / 2}$

backscattering cross section of one bubble

backscattering cross section of $N$-bubble array

target strength $\left(=10 \log \left|f_{\mathrm{bs}}\right|^{2}\right)$

transmission coefficients for transmission from medium " 1 ", to " 2 "' or " 2 "' to " 1 ," respectively $\left[T_{i j}=2\left(\rho_{j} c_{j} / \rho_{i} c_{i}\right) /\left(1+\left(\rho_{j} c_{j} / \rho_{i} c_{i}\right)\right)\right]$

angle of orientation of body relative to the direction of the incident wave ( $\theta=0$ corresponds to broadside incidence)

launch/land angle for Lamb wave

volume of body

deviation of radius from mean radius of irregular sphere at a given point on sphere subscripts indicating medium " 1 " (surrounding fluid) and medium ' 2 "' (body medium)

average over ensemble of statistically independent samples

\section{INTRODUCTION}

In the first part of this series of papers, laboratory data showed that the scattering properties of zooplankton from different gross anatomical groups varied between the groups (Stanton et al., 1997). In this paper, the acoustic boundary conditions inferred from that study are used to derive approximate scattering models of single echoes from an individual animal, the ping-to-ping variability of the echoes as the animal changes shape and orientation, as well as the average echo value. The models are compared with the laboratory data.

The single ping data and variability analyses in this twopart study are most revealing of the fundamental physics of the scattering processes and serve at least as a guide toward development of the models. In addition, those analyses are useful when examining resolved echoes in the field. The analysis involving average echoes is less revealing of the fundamental scattering process, but is useful in modeling of volume reverberation in field surveys.

\section{SCATTERING MODELS}

\section{A. Euphausiids (deformed fluid cylinder)}

In Stanton et al. (1998), our analysis of data acquired in at-sea laboratory studies indicated that there were typically at least two arrivals from the body of the animal. We hypothesized that, near broadside incidence, there are two major arrivals, one from the front interface of the body and the other from the back interface (after the incident wave has traveled into the body) (Fig. 1). For a weakly scattering body (i.e., one in which the density and sound speed of the body are close to that of the surrounding medium), the wave that travels into the body suffers little transmission loss at the

front boundary. As a result, once reflected off the back interface, it can be of comparable strength to that of the echo from the front interface. The strong regular interference patterns of target strength versus frequency imply that the two waves are indeed of comparable strength and that the animal can be considered as a weakly scattering body. It was also hypothesized that for other orientations (or even irregular shapes at broadside), other parts of the body may also contribute significantly to the scattering, giving rise to six or more echoes from the body. As a result, many of the target strength versus frequency curves had an irregular structure.

The scattering by this type of animal is quite complex as its shape resembles that of a deformed finite length cylinder, a shape for which there is no exact analytical solution. The choice of which approach to use in modeling the scattering depends upon the application. If the emphasis is on the structure of the target strength versus frequency curves for individual ping data, then enough detail of the boundaries must be included so that the interference patterns can be predicted (at least qualitatively). However, if only averages over many pings and animals are of interest, then the structure of the interference pattern is reduced as a result of the average and a simpler model can be considered.

There has been much development to date on description of scattering by weakly scattering finite-length fluid cylinders which involves a modal-series-based line integral (deformed cylinder formulation), a ray summation (derivable from surface integrals or Sommerfeld-Watson transformation imbedded in deformed cylinder formulation), and a distorted wave Born approximation (DWBA, a volume integral which can be reduced to a line integral in this case) (Stanton, 1988, 1989a, 1989b; Stanton et al., 1993a, 1993b, 1994a, 1994b; Chu et al., 1992, 1993). The DWBA approach has been very useful in calculating (through numerical integra- 
tion) average echoes from animals of arbitrary distributions of sizes and orientation angles. The ray formulation has been especially useful in both qualitatively illustrating the structure of target strength versus frequency curves as well as being easy to manipulate in deriving simple (yet accurate) closed-form expressions for echoes averaged over a constrained set of distributions of size and orientation angle.

The DWBA formulation is given in a general volume integral form for backscattering in the farfield due to a finitelength body as (Morse and Ingard, 1968)

$$
f_{\mathrm{bs}}=\frac{k_{1}^{2}}{4 \pi} \iiint_{v}\left(\gamma_{\kappa}-\gamma_{\rho}\right) e^{2 i\left(\mathbf{k}_{i}\right)_{2} \cdot \mathbf{r}_{v}} d v,
$$

where the material property parameters are expressed in terms of compressibility $(\kappa)$ and density $(\rho)$ as

$$
\begin{aligned}
& \gamma_{\kappa}=\left(\kappa_{2}-\kappa_{1}\right) / \kappa_{1}, \\
& \gamma_{\rho}=\left(\rho_{2}-\rho_{1}\right) / \rho_{2},
\end{aligned}
$$

where medium " 1 " (indicated by a subscript) is the surrounding water and medium " 2 " (also indicated by a subscript) is the body of the animal. Also, the subscript " 2 " to $\rho$ in the denominator in Eq. (3) represents a correction to the subscript "1" given in Chu et al. (1993) and Stanton et al. (1993b). Given that these are weakly scattering bodies and $\rho_{2} \simeq \rho_{1}$, the difference is negligible. The compressibility is written in terms of density and sound speed as

$$
\kappa=\left(\rho c^{2}\right)^{-1} .
$$

In this formulation, the material property terms are allowed to vary inside the volume. Note that this formula is the complex conjugate of the one presented in Morse and Ingard and is consistent with the phase shift convention of $e^{+i k r}$ for an outgoing scattered wave. Also, in this "distorted wave" formulation, the incident wave number in the exponent is evaluated inside the medium $\left[\left(\mathbf{k}_{i}\right)_{2}\right]$. That is, the wave number of the wave traveling inside the body has a magnitude $k_{2}$. There will be phase errors associated with this distorted wave formulation for conditions under which the incident ray will pass through part of the body, into the surrounding medium, and back into the body again (such as in the case of end-on incidence for a bent cylinder). Correction for the phase shift for the path in the water can, in principle, be taken into account through piecewise integration. For an object whose cross section is circular at every point along the lengthwise axis (i.e., a deformed circular cylinder), two dimensions of the integration (within a cross section at an arbitrary point along the cylinder) can be performed analytically, which reduces the formulation to a line integral:

$$
\begin{aligned}
f_{\mathrm{bs}}= & \frac{k_{1}}{4} \int_{\mathbf{r}_{\mathrm{pos}}}\left(\gamma_{\kappa}-\gamma_{\rho}\right) e^{2 i\left(\mathbf{k}_{i}\right)_{2} \cdot \mathbf{r}_{\mathrm{pos}}} \\
& \times a \frac{J_{1}\left(2 k_{2} a \cos \beta_{\text {tilt }}\right)}{\cos \beta_{\text {tilt }}}\left|d \mathbf{r}_{\mathrm{pos}}\right|,
\end{aligned}
$$

where now the material properties are allowed to vary with respect to position along the lengthwise axis, but restricted to remaining constant within each infinitesimally thin cross section at any given point along that axis. In this equation, $\gamma_{\kappa}$, (a)

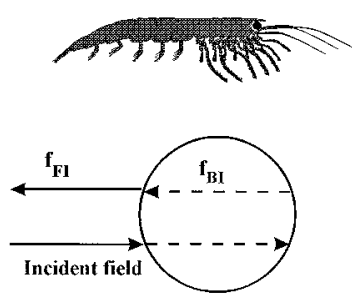

(b)

(c)

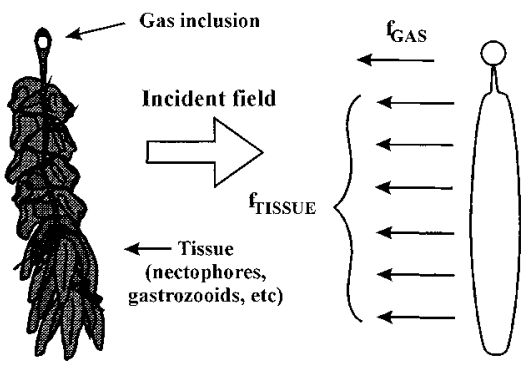

FIG. 1. Zooplankton and corresponding illustrations of certain important scattering components.

$\gamma_{\rho}, a$, and $\beta_{\text {tilt }}$ can be functions of $\mathbf{r}_{\mathrm{pos}}$. The term $J_{1}$ is the Bessel function of the first kind of order 1 .

This DWBA-based deformed cylinder formulation is similar in form to the modal-series-based deformed cylinder formulation presented in Stanton (1989a). The differences lie in the fact that the DWBA is only accurate for weakly scattering bodies while the modal-series-based solution can describe a wide range of (axisymmetric) material profiles (elastic shelled body, etc.). However, the DWBA formulation, by the nature of its volume integration, is accurate for all angles of orientation, while the modal-series-based solution is only accurate near broadside incidence because it uses modalseries coefficients from an infinitely long cylinder.

For a uniformly bent cylinder, the term in the exponent $\left(\mathbf{k}_{i}\right)_{2} \cdot \mathbf{r}_{\mathrm{pos}}=k_{2} \rho_{c}\left(1-\cos \beta_{\mathrm{tilt}}\right)$. Using $\left|d \mathbf{r}_{\mathrm{pos}}\right|=\rho_{c} d \beta_{\text {tilt }}$ gives the integral expression

$$
\begin{aligned}
f_{\mathrm{bs}}= & \frac{k_{1} \rho_{c}}{4} e^{i 2 k_{2} \rho_{c}} \int\left(\gamma_{\kappa}-\gamma_{\rho}\right) e^{-i 2 k_{2} \rho_{c} \cos \beta_{\mathrm{tilt}}} \\
& \times \frac{a J_{1}\left(2 k_{2} a \cos \beta_{\mathrm{tilt}}\right)}{\cos \beta_{\mathrm{tilt}}} d \beta_{\mathrm{tilt}} .
\end{aligned}
$$

This expression is accurate for all angles of orientation and arbitrary variability of the cross-sectional radius along the length of the body (such as a tapered cylinder). The cylinder is bent in the plane containing $\mathbf{k}_{i}$. For broadside incidence (i.e., the body is bent symmetrically away from the echo sounder), the integral is symmetrical about $\beta_{\text {tilt }}=0$. For end-on incidence, the integral is symmetrical about $\beta_{\text {tilt }}$ $=\pi / 2$.

For broadside incidence, the integral can be performed using the method of stationary phase, 


$$
f_{\mathrm{bs}} \simeq \frac{k_{1} a}{4 \sqrt{2}}\left(\gamma_{\kappa}-\gamma_{\rho}\right) \sqrt{\rho_{c} \lambda_{1}} J_{1}\left(2 k_{2} a\right) e^{i \pi / 4}
$$

where the condition $2 k_{2} \rho_{c}\left(1-\cos \left(\beta_{\mathrm{tilt}}\right)_{\max }\right) \gg 1$ was required in order to use the method of stationary phase. This condition requires the body to be bent enough so that many Fresnel zones are present on it (this corresponds to having deflection of the end points much greater than an acoustic wavelength).

Limiting expressions (with respect to wavelength) of the scattering can be determined from both Eqs. (1) and (7). At very low values of $k a$ and wavelengths long enough so that the bend does not affect the scattering, Eq. (1) can be evaluated in the long wavelength limit. For shorter wavelengths, there are two other regions of scattering: (i) $k a \ll 1$ with the condition that the wavelength is short enough so that the bend does affect the scattering and (ii) $k a \gg 1$. For these latter two cases, Eq. (7) can be evaluated by inserting the appropriate limiting expression for the Bessel function. Using Eqs. (1) and (7) as indicated above, the scattering limits for broadside incidence are given by

$$
\begin{aligned}
f_{\mathrm{bs}}= & \frac{\left(k_{1} a\right)^{2}}{4} L\left(\gamma_{\kappa}-\gamma_{\rho}\right), \\
& k a \ll 1 \quad \text { and } 2 k \rho_{c}\left(1-\cos \left(\beta_{\mathrm{titl}}\right)_{\max }\right) \ll 1, \\
f_{\mathrm{bs}}= & \frac{\left(k_{1} a\right)^{2}}{4 \sqrt{2}} \sqrt{\rho_{c} \lambda_{1}}\left(\gamma_{\kappa}-\gamma_{\rho}\right) e^{i \pi / 4}, \\
& k a \ll 1 \quad \text { and } 2 k \rho_{c}\left(1-\cos \left(\beta_{\mathrm{tilt}}\right)_{\max }\right) \gg 1, \\
f_{\mathrm{bs}}= & \frac{\sqrt{\rho_{c} a}}{4}\left(\gamma_{\kappa}-\gamma_{\rho}\right) \cos \left(2 k_{2} a-\frac{3}{4} \pi\right) e^{i \pi / 4}, \\
& k a \gg 1 \quad \text { and } 2 k \rho_{c}\left(1-\cos \left(\beta_{\mathrm{tilt}}\right)_{\max }\right) \gg 1, \\
= & -\frac{\sqrt{\rho_{c} a}}{8}\left(\gamma_{\kappa}-\gamma_{\rho}\right) e^{-i 2 k_{1} a}\left(1+i e^{i 4 k_{2} a}\right)
\end{aligned}
$$

(ray form).

The approximation $k_{2} \simeq k_{1} \simeq k$ was used, where appropriate, in the above equations. Typically, it is important to distinguish between $k_{1}$ and $k_{2}$ in phase shift terms but not in amplitude terms. Phase terms such as the exponent in $e^{i 4 k_{2} a}$ greatly influence the position of the nulls in the target strength versus frequency curves. Equation (11) is equivalent to Eq. (10) and is written to illustrate arrivals from the front and back interface of the body (first and second term within the parentheses, respectively). For $k a \ll 1$ and $k L \ll 1$, Eq. (8) applies for all angles of orientation.

While these limiting expressions at broadside incidence are very useful, the orientation dependence of the scattering (for all $\mathrm{ka}$ ) generally must be determined through numerical integration of Eq. (6). Predicting volume scattering strengths due to aggregations of animals in the ocean involves numerically calculating averages over angle of orientation and size. In this case, structure in the target strength versus frequency curves for single echoes from individuals will be greatly reduced. The structure is quite sensitive to the precise size, shape, orientation, and material properties of the animal.
Since this structure is greatly reduced or washed out in an average, these parameters do not need to be known as accurately for the predictions. However, when the structure of the curves of single resolved echoes is required for analysis, the boundary must be made more realistic, which tends to make the problem more complex. It can be taken into account either by directly incorporating a complex, realistic boundary into the integral in Eq. (1) or by direct summation of echoes from various parts of the body. Use of Eq. (1) is far more precise (providing that the actual boundary is known precisely); however, summation of echoes in an approximate manner has utility as it can provide the statistical properties of the echoes quite readily. Analytical manipulation of the sum can also be done more readily than with the general integral formula.

A general high-frequency (geometric scattering region) ray formula which adds an arbitrary number of $(N)$ "glint" rays together to produce the total echo of the animal can be written as

$$
f_{\mathrm{bs}} \simeq \sum_{j=1}^{N} b_{j} e^{i 2 k_{1} \epsilon_{j}},
$$

where the (complex) amplitude coefficient $b_{j}$ is given for two common cases of spherical curvature as follows:

$$
\begin{aligned}
b_{j} \simeq & \frac{1}{2} \mathscr{B}_{12} a \quad \text { (facing interface, convex } \\
& \text { from viewpoint of source/receiver), }
\end{aligned}
$$

$$
b_{j} \simeq-\frac{1}{2} T_{12} T_{21} \mathscr{B}_{21} a
$$

(back interface, concave from viewpoint of source/receiver).

The phase shift of each ray due to the relative location of the interface from which it scatters is taken into account in the exponent where $\epsilon_{j}$ is the deviation of the point of scatter from a (zero phase) reference plane that is normal to the direction of the incident acoustic wave. The term $\epsilon_{j}$ is negative for points on the source/receiver side of the reference plane. For a sphere whose center is on the zero phase plane, $\epsilon_{j}=-a$ and $+a$ for the front interface [Eq. (13)] and back interface [Eq. (14)] cases, respectively. The reflection coefficients of the front and back interfaces have been taken into account in $b_{j}$ (note that $\mathscr{B}_{21}=-\mathscr{B}_{12}$ ). The plane tangent to the middle of each curved section is perpendicular to the direction of incidence giving rise to the glint (i.e., the sections are broadside to the transducers). For surfaces with more complex curvature such as the side of a bent cylinder, then $b_{j}$ is more complex. For example, for a convex interface described in planes "(1)" and "(2)" by two local radii of curvature $\left(\rho_{j}^{(1)}, \rho_{j}^{(2)}\right), a$ is replaced by $\left(\rho_{j}^{(1)} \rho_{j}^{(2)}\right)^{1 / 2}$ (Gaunaurd, 1985). The phase of $b_{j}$ depends specifically upon the curvature (convex, concave, cylindrical, spherical, compound, etc.). For example, the phase for the concave spherical surface is $\pi$ as indicated by the minus sign in Eq. (14). The wave number is held fixed at $k_{1}$ in this formulation for simplicity. As a result, the position of the nulls in any TS versus frequency curve would be slightly in error. The simplification does not significantly affect the results, especially 
when averages or statistics of random phase $\left(2 k_{1} \epsilon_{j}\right)$ ensembles are analyzed.

This formula is written down heuristically based upon various other formulations. Great success has been achieved with a two-ray formulation (Stanton et al., 1993a, 1993b) in which the rays only from the front and back interfaces at broadside incidence are taken into account. In the two-ray formulation, the local reflection and transmission coefficients were taken into account as well as the phase shifts, radius of curvature of the bent axis, and cylindrical radius of the cross section. A six-ray model in which each local scatterer had associated a random phase and unity amplitude was used in Stanton et al. (1994b) to describe the statistical properties of the scattering by a decapod shrimp off broadside incidence (more than six rays gave the same statistical behavior of the echo envelope, so the summation was truncated to include only six rays).

The two-ray version of Eq. (12) has experienced much development and use. In Stanton et al. (1993a, 1993b), an approximate two-ray model was derived for all angles of incidence:

$$
f_{\mathrm{bs}} \simeq \frac{1}{2} \sqrt{\rho_{c} a} \mathscr{R}_{12} e^{-i 2 k_{1} a} I_{0} e^{-\alpha_{B}\left(2 \theta \rho_{c} / L\right)^{2}},
$$

where

$$
I_{0}=1-T_{12} T_{21} e^{i 4 k_{2} a} e^{i \mu\left(k_{1} a\right)} .
$$

This expression for scattering amplitude was shown to depend upon the radius of curvature $\rho_{c}$ of the longitudinal axis of the body, radius $a$ of the cross section of the body, reflection coefficient $\mathscr{B}_{12}$ of the front interface, length $L$, and angle of orientation $\theta$. The width of the main lobe of the scatter versus angle pattern based on this formula best fit more precise DWBA-based calculations when the parameter $\alpha_{B}=0.8$. In order for this ray solution to be valid for values of $k_{1} a$ less than unity, the following phase shift term was used (Stanton et al., 1993a):

$$
\mu\left(k_{1} a\right) \simeq \frac{-(\pi / 2) k_{1} a}{k_{1} a+0.4} .
$$

As a result, the scattering amplitude is valid for values of $k_{1} a$ as low as 0.1 . For orientations far away from broadside incidence, more sophisticated models, such as the DWBA, must be used and evaluated numerically. However, the above equation for the scattering amplitude works well for single echoes near broadside or can be used in accurately averaging over angle of orientation over a wide range of angles (the inaccuracies far from broadside incidence are not significant provided the average includes contributions from near broadside incidence where the scattering is the strongest).

The above expression for backscattering amplitude can be used to average the backscattering cross section over an arbitrary range of length and angle of orientation provided the average includes broadside incidence. A convenient formula was derived in Stanton et al. (1993b) for an average over a narrow range of animal sizes:

$$
\left\langle\sigma_{\mathrm{bs}}\right\rangle_{\theta, L} / \overline{L^{2}}=A_{i j} \mathscr{B}_{12}^{2}\left\langle\left|I_{0}\right|^{2}\right\rangle_{L} \beta^{-1},
$$

where $\bar{L}$ is the mean of the narrow length distribution (and narrow in this case means the width of the distribution is much less than the mean length). For a narrow distribution, $\left\langle\left|I_{0}\right|^{2}\right\rangle_{L}=2\left\{1-\exp \left[-8(k \overline{a s})^{2}\right] \cos (4 k \bar{a}+\mu)\right\}$, where $s$ is the standard deviation of length, normalized by the mean length. The term $A_{i j}$ was determined in that paper for all four combinations of straight and bent cylinders, and Gaussian and uniform $(0-2 \pi)$ distributions of angle of orientation. For a bent cylinder whose angle of orientation is Gaussian distributed, $A_{i j}$ is given as

$$
A_{i j}=T_{B}^{2} C_{B}^{2} /\left(16 \sqrt{\alpha_{B}} s_{\theta}\right),
$$

where $s_{\theta}$ is the standard deviation of orientation distribution (in radians), and $T_{B}$ and $C_{B}$ are empirically determined parameters from simulations using the DWBA $\left(T_{B}=1, C_{B}\right.$ $=1.2$ ).

\section{B. Gastropods (deformed elastic-shelled sphere)}

The backscatter data from gastropods presented in Stanton et al. (1998) showed overall (high) echo levels consistent with that of a dense and/or hard scatterer. Furthermore, the data indicate the possibility of an echo from part of the body traveling at a subsonic speed and then interfering with the echo from the front interface (Fig. 1). These properties of the echoes are consistent with the fact that the outer boundary of the animal is a dense, hard elastic shell made of aragonite. The subsonic wave is possibly a flexural Lamb wave that circumnavigates the body at a subsonic speed before returning to the transducer (see, for example, Kargl and Marston, 1989; Kaduchak et al., 1995).

Modelling the scattering of sound by gastropods is a great challenge because of the complexity of the boundary. The shell is irregular and contains a major discontinuity (the opercular opening). Our attempts (not shown) to model it as an idealized spherical shell using the exact formulation of Goodman and Stern (1962) (and taking into account differences between the interior tissue and surrounding fluid) were unsuccessful, even as a first approximation. The idealized sphere model produced resonances not seen in the data (some of the broader resonances remained even after averaging over a distribution of sizes). Therefore, a more realistic model must be used. Rigorous treatment of this problem would include numerical evaluation of the wave equation (Jansson, 1993) or a sophisticated generalized ray theory (Felsen and Lu, 1989; Ho and Felsen, 1990; Norris and Rebinsky, 1994; Ho, 1994; Yang et al., 1995; Rebinsky and Norris, 1995, and Yang et al., 1996).

In order to describe the scattering by such an irregular body, we chose a (analytical) ray approach over a numerical one because certain ray models can not only provide greater insight into the physics of the scattering process, but can also be manipulated algebraically for other calculations. The ray formulation in the case of the weakly scattering body described above [Eqs. (12)-(19)] involved rays scattering from the front and back interfaces of the body. However, with the hard elastic shell of the gastropod, the incident ray cannot penetrate the shell with much energy. In contrast, (circumferential) shell waves are excited by the incident field and travel around the shell, continuously shedding off energy. Some of this energy sheds or "leaks" back toward the sound 
source/receiver and will interfere with the ray that is reflected off the front portion of the shell. As a result, the scattering amplitude versus frequency plots have peaks and dips corresponding to the constructive and destructive interference, respectively.

Given the complexity of the problem of scattering by an irregular shell, we have found it convenient to begin with the ray formulation for an idealized spherical shell, and then heuristically modify the formulation to take into account roughness and discontinuities. The result is an approximate formula that illustrates effects due to those features. We begin with a ray formulation from Kargl and Marston (1989) and summarized in Marston et al. (1990) and Marston (1992). It gives the usual decomposition of the scattered wave into various components:

$$
f_{\mathrm{bs}} \simeq f_{\mathrm{spec}}+f_{\mathrm{tw}}+f_{\mathrm{Lamb}}+f_{\mathrm{Franz}},
$$

where the $f_{\text {spec }}$ or specular term contains specularly "reflected" echoes from the front interface, and $f_{\text {tw }}$ corresponds to all internal refractions and reflections within the shell and interior fluid. The term $f_{\text {Lamb }}$ contains the summation of the different classes of Lamb waves, antisymmetric and symmetric of all order, each of which involves the superposition of all singly and multiply circumnavigated waves. The last term is due to the Franz wave. The Lamb and Franz waves are both circumferential waves - the Lamb or "plate", waves represent various classes of plate deformations that circumnavigate the body in a wavelike manner while continuously leaking energy into the surrounding fluid. The Franz or "creeping", waves travel along the boundary but within the surrounding fluid.

Evaluation of each term in Eq. (20) is quite involved. The backscattering data involving gastropods indicate the presence of two major echoes coming from each animal. We assume that one of the echoes is from the front interface of the animal. The other echo has the strength and (subsonic) speed consistent with that of Lamb waves under certain conditions. In order to model the scattering by those animals and for simplicity in the analysis, we retain only the (hypothesized) dominant terms for the case of hard, dense spherical elastic shells, $f_{\text {spec }}$ and $f_{\text {Lamb }}$ :

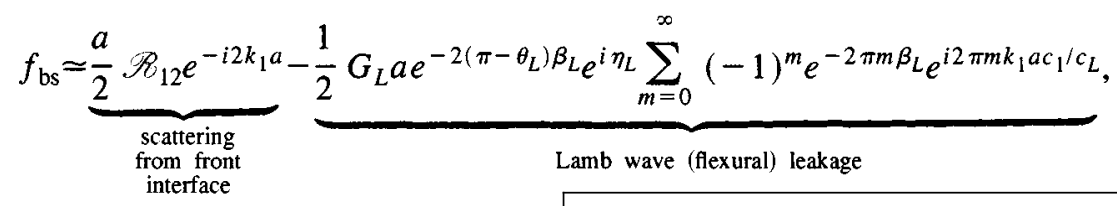

where

$$
\begin{aligned}
& \eta_{L}=2 k_{1} a\left[\left(c_{1} / c_{L}\right)\left(\pi-\theta_{L}\right)-\cos \theta_{L}\right]-\pi / 2, \\
& \theta_{L}=\sin ^{-1}\left(c_{1} / c_{L}\right), \\
& G_{L} \simeq 8 \pi \beta_{L} c_{1} / c_{L},
\end{aligned}
$$

and

$$
c_{L} / c_{1} \simeq k_{1} a /\left(\alpha_{L}+1 / 2\right) \text {. }
$$

The two terms in the expression for the scattering amplitude correspond to the scattering from the front portion of the shell and the lowest-order antisymmetric Lamb wave (flexural wave) that circumnavigates the shell $m$ times, respectively $[m=0$ corresponds to traveling around the shell an amount $2\left(\pi-\theta_{L}\right)$ radians, $m=1$ corresponds to traveling around the shell an amount $2\left(\pi-\theta_{L}\right)+2 \pi$, etc.] where $\theta_{L}$ is the angle at which the Lamb wave lands onto and launches from the shell (for subsonic waves, $\theta_{L}=\pi / 2$ ). The term $\eta_{L}$ is a phase shift term corresponding to the phase shift incurred on the shell by the $m=0$ Lamb wave (this travel-path and caustic-related phase is relative to the zero-phase reference plane that contains the center of the sphere and is perpendicular to the direction of the incident wave), $G_{L}$ is the combined coupling coefficient for the conversion of the fluidborne sound into the Lamb wave and back into fluid-borne sound, $c_{L}$ is the speed of the Lamb wave, and $\alpha_{L}$ and $\beta_{L}$ are the real and imaginary parts of the complex root $\nu_{L}$ of the denominator of the modal series coefficient (not shown) for a fluid-filled spherical elastic shell $\left(\beta_{L}\right.$ is the attenuation coefficient of the Lamb wave due to its continuous shedding or leaking of energy into the surrounding fluid) (Marston, 1992). Here, $c_{L}, \alpha_{L}$, and $\beta_{L}$ are generally complicated functions of $k a$.

The above expression describing the scattering by a shell is approximate as it only takes into account one class of Lamb wave (other Lamb waves could quite readily be taken into account by simply summing over other ' $L$ ' 'type indices, however the zeroth-order antisymmetric Lamb wave tends to dominate the scattering for the low-to-moderate values of $k a$ in our data sets and is the only wave being included in this analysis (Kargl and Marston, 1989). Furthermore, the thickness resonance is ignored. This resonance is due to the front ray penetrating the outer boundary of the shell and experiencing multiple internal reflections within the shell material (for the hard shell of the gastropod, it is assumed that little energy penetrates the outer boundary). For similar reasons, internal refractions and reflections within the body interior are ignored. Exact values of the terms $G_{L}, c_{L}$, $\beta_{L}$, and $\alpha_{L}$ must be obtained through numerical evaluation of the Sommerfeld-Watson transformation of the modal series solution and is beyond the scope of this present analysis (Kargl and Marston, 1989).

As previously discussed, one major complication of the modeling for the gastropod involves the fact that the body is not perfectly spherical but irregular. In order to estimate effects of roughness on the scattering, the above approximate 
ray-based formulation is heuristically modified. The radius of the body at a given point on the body is written as a stochastic parameter,

$$
a=\bar{a}+\zeta,
$$

where $\bar{a}$ is the mean radius and $\zeta$ is the random deviation of the radius from the mean at a given point on the shell. The Lamb wave will travel many different paths resembling meridional lines (not shown in the two-dimensional plot in Fig. 1). Because of the boundary deformations, the paths will randomly deviate from pure meridional lines. Each path will experience variations in local radii of curvature resulting in a slightly different effective radius $\bar{a}+\Delta_{a}$ for that path [where the effective radius is the circumference (along a rough path) divided by $2 \pi$ ]. The phase shift of the differential portion of the Lamb wave that travels along a given path will vary depending upon the particular path taken.

In order to estimate the effects of roughness on the total Lamb wave, we use Eq. (21) to heuristically write an approximate expression for the differential Lamb wave that travels within a differential meridional angle:

$$
\begin{aligned}
d f_{\mathrm{Lamb}} \simeq & -\frac{1}{2} G_{L} a e^{-2\left(\pi-\theta_{L}\right) \beta_{L}} e^{i \eta_{L}} \sum_{m=0}^{\infty}(-1)^{m} \\
& \times e^{-2 \pi m \beta_{L}} e^{i 2 \pi m k_{1} a c_{1} / c_{L}} \frac{d \phi_{M}}{2 \pi},
\end{aligned}
$$

where $\phi_{M}$ is the meridional angle. The total Lamb wave is calculated by integrating the above expression over all meridional angles:

$$
\begin{aligned}
f_{\mathrm{Lamb}}= & -\frac{1}{2} \int_{0}^{2 \pi} G_{L} a e^{-2\left(\pi-\theta_{L}\right) \beta_{L}} e^{i \eta_{L}} \\
& \times \sum_{m=0}^{\infty}(-1)^{m} e^{-2 \pi m \beta_{L}} e^{i 2 \pi m k_{1} a c_{1} / c_{L}} \frac{d \phi_{M}}{2 \pi},
\end{aligned}
$$

where now $a$ is the effective radius, as defined above ( $a$ $=\bar{a}+\Delta_{a}$ ), for a given meridional angle. Note that, given the symmetry of the scattering geometry, only half of the range of angles contains a unique set of effective radii. For a perfectly smooth ideal sphere, the integrand is constant with respect to the meridional angle and the integral reduces to the Lamb wave component of Eq. (21). If $a$ is randomly distributed over the $0-2 \pi$ range of meridional angles, then this integral is roughly equivalent to the ensemble average over the distribution of $\Delta_{a}$ :

$$
\begin{aligned}
f_{\text {Lamb }} \simeq & \left\langle-\frac{1}{2} G_{L} a e^{-2\left(\pi-\theta_{L}\right) \beta_{L}} e^{i \eta_{L}}\right. \\
& \left.\times \sum_{m=0}^{\infty}(-1)^{m} e^{-2 \pi m \beta_{L}} e^{i 2 \pi m k_{1} a c_{1} / c_{L}}\right\rangle .
\end{aligned}
$$

This equivalency is analogous to the ergodic theorem where a temporal average of a quantity is equal (under certain conditions) to the ensemble spatial average of that quantity (Skudrzyk, 1971). In this analysis, $\phi_{M}$ replaces time. The analogy to the ergodic theorem is not perfect as the temporal average in the theorem is taken over the limit of all time whereas the integral over $\phi_{M}$ involves a finite range of $\phi_{M}$.

Using the fact that the complex phase shift terms in this average are more important in this average than the random (real) amplitude terms, only the random complex exponential terms will be treated in this average (Stanton, 1992). For mathematical convenience and given the fact that many random natural processes tend to follow Gaussian statistics, $\Delta_{a}$ is assumed to be Gaussian distributed and the formula

$$
\left\langle e^{i \gamma \Delta a}\right\rangle=e^{-(1 / 2) \gamma^{2} \sigma^{2}}
$$

is used to obtain the following approximate expression:

$$
\begin{aligned}
f_{\mathrm{bs}} \simeq & \frac{a}{2} \mathscr{B}_{12} F_{\mathrm{spec}} e^{-i 2 k_{1} a}-\frac{1}{2} G_{L} e^{i \Phi_{L}} \bar{a} e^{-2\left(\pi-\theta_{L}\right) \beta_{L}} \\
& \times e^{i 2 k_{1} a\left[\left(c_{1} / \bar{c}_{L}\right)\left(\pi-\theta_{L}\right)-\cos \theta_{L}\right]-i \pi / 2} F_{L} \\
& \times \sum_{m=0}^{\infty}(-1)^{m} e^{-2 \pi m \beta_{L}} e^{i 2 \pi m k_{1} \bar{a}_{1} / \bar{c}_{L}} e^{-(1 / 2) \gamma^{2} \sigma^{2}},
\end{aligned}
$$

where

$$
\begin{aligned}
\gamma= & k_{1}\left\{2\left[\left(c_{1} / \overline{c_{L}}\right)\left(\pi-\theta_{L}\right)-\cos \theta_{L}\right]+2 \pi m c_{1} / \bar{c}_{L}\right. \\
& \left.+B k_{1} \bar{a}\left[2\left(\pi-\theta_{L}\right)+2 \pi m\right]\right\} .
\end{aligned}
$$

This formula takes into account dispersion of the Lamb wave by linearizing the dependence of $c_{1} / c_{L}$ upon $k_{1} a$ over the narrow range of values of $k a$ as $k \bar{a}$ is fixed and $\Delta_{a}$ is varied. Here $c_{1} / c_{L}=c_{1} / \bar{c}_{L}+B k_{1} \Delta_{a}$, where $\bar{c}_{L}$ is the value of $c_{L}$ evaluated at $k \bar{a}$. Dispersion of the flexural wave is much stronger for lower values of $k a$ (e.g., $k a<20)$ than for higher values (Kargl and Marston, 1989). The angle $\theta_{L}$ stays fixed at $\pi / 2$ during the averaging provided $c_{L}$ remains subsonic.

While the derivation of Eq. (31) was far from rigorous, these results show that the Lamb wave term in the scattering amplitude becomes attenuated due to the randomness of the phase of the various Lamb wavelets traveling around the irregular body. In addition to taking into account the randomness of the irregularities, three other terms were incorporated heuristically: (1) the term $F_{\text {spec }}$ to take into account a reduction of echo level from the front interface for orientations in which part or all of the opercular opening is facing the echosounder $\left(F_{\mathrm{spec}}=1\right.$ when the opening is facing away from the sounder, $0 \leqslant F_{\text {spec }}<1$ when part or all of the opening is facing the sounder). (2) The term $F_{L}$ takes into account the fact that part of the Lamb wave does not travel beyond the opening that it may encounter $\left(F_{L}=0\right.$ when the opening faces away from the echosounder and no Lamb waves can propagate beyond the opening, $0<F_{L} \leqslant 1$ for other angles of orientation). This term must be determined strictly from geometrical arguments. Reflections of the Lamb wave off the discontinuity are ignored. (3) Because of irregularities and uncertainties in material properties, the term $e^{i \Phi_{L}}$ was inserted to account for any deviation in phase shift from that predicted from an idealized theory. The $a$ in the first term represents the local radius of curvature of the shell surface seen by the acoustic source/receiver. 


\section{Siphonophore (bubble plus tissue)}

The backscatter data from siphonophores presented in Stanton et al. (1998) indicated that the gas inclusion in the pneumatophore dominated the overall average levels of the echoes. However, the tissue surrounding the gas sometimes significantly contributed to the pattern of the target strength versus frequency curves. The scattering amplitude can be written in terms of the separate contributions of the scattering from the two parts of the body (Fig. 1):

$$
f_{\text {bs }}=f_{\text {bubble }}+f_{\text {tissue }} \text {. }
$$

This formulation assumes that shadowing from each component does not significantly affect the scattering by the other. This is a reasonable assumption since the tissue is most likely a weakly scattering material and the incident acoustic wave travels through it essentially unperturbed. Since the gas inclusion is so small, the shadow region behind it is much smaller than the total volume of the tissue and hence little of the scattering by the tissue will be affected.

Prediction of the total echo from the siphonophore requires evaluation of each of the above components of the scattered field. The following exact formula is used to calculate the scattering by the pneumatophore:

$$
\begin{aligned}
f_{\text {bubble }}= & \frac{-i}{k_{1}} \sum_{m=0}^{\infty}(2 m+1) \\
& \times(-1)^{m} b_{m}^{(f)} \quad \text { exact, all } k_{1} a .
\end{aligned}
$$

This formula was taken directly from Anderson (1950). It is an exact expression for the scattering of sound by a fluid sphere (gas behaves acoustically as a fluid as it does not support shear waves). The gas inclusion in siphonophores may depart from being a perfect sphere, hence this equation should be considered to be an approximation to the scattering by the bubble. The term $b_{m}^{(f)}$ is the modal series coefficient for a fluid sphere.

Evaluation of the scattering by the tissue is a great challenge. If one were to choose a simple boundary like a smooth bent cylinder, the statistical nature of the structure of the target strength versus frequency curve may not be accurately predicted. However, the boundary of the siphonophore is sometimes quite complex, and it may not be possible to produce a reasonable mathematical construction of the outer boundary. A general approach in estimating the scattering by the tissue would be to use the general volume integral of the DWBA. Adding that contribution to the one from the gas gives the backscattering amplitude from the entire animal:

$$
\begin{aligned}
f_{\mathrm{bs}} \simeq & -\underbrace{\frac{i}{k_{1}} \sum_{m=0}^{\infty}(2 m+1)(-1)^{m} b_{m}^{(f)}}_{\text {gas inclusion }} \\
& +\underbrace{\frac{k_{1}^{2}}{4 \pi} \iiint_{v}\left(\gamma_{\kappa}-\gamma_{\rho}\right) e^{2 i\left(\mathbf{k}_{i}\right)_{2} \cdot \mathbf{r}_{v}} d v}_{\text {tissue }},
\end{aligned}
$$

where the terms of the integral are defined earlier in this paper. This approach allows one to construct a complex mathematical boundary describing the body of the siphonophore and perform the integration directly. In order for the statistics of the target strength versus frequency curve to be determined, the boundary must be randomly perturbed (changing shape and orientation, etc.) over an ensemble of pings. Such a procedure, while rigorous, would be quite tedious.

Another approach to obtain echo statistics can involve the heuristic ray formulation described earlier in this paper. With that approach, only the contributions from various major scattering features of the body are included in a ray summation. A major feature in this context is defined as a facet or facetlike part of the body that is broadside to the incident beam (giving rise to "glint"). This feature would scatter the sound back toward the receiver with a level much greater than that of a feature that is not broadside. With this ray approach, the echo from the whole animal is

$$
f_{\mathrm{bs}} \simeq-\frac{i}{k_{1}} \sum_{m=0}^{\infty}(2 m+1)(-1)^{m} b_{m}^{(f)}+\sum_{j=1}^{N} b_{j} e^{i 2 k_{1} \epsilon_{j}}
$$

There is great utility to this approach. Modal-series component: This exact solution to a spherical bubble will predict echo levels that are accurate for all values of $k a$. For the siphonophores in which the gas dominates the average echo level at all frequencies, use of the expression can produce reasonable estimates of the average target strength of the siphonophores. Ray component: (1) It is simple in form and can be readily manipulated and evaluated. (2) For six or more random-phase rays, the statistical nature or probability density function (PDF) of this ray component will be Rayleigh-PDF-like. Once the ray component is added to the modal series component, the statistics of the envelope of the total echo will be Ricean. As a practical matter, it may be difficult to directly determine the values of $b_{j}$. If data are available on a particular siphonophore, it is reasonable to adjust the values of $b_{j}$ according to the observed contribution from the tissue.

Analytical averaging of the predicted backscattering cross section from the siphonophore over a range of orientation angles, in general, involves many cross terms, and it would be more practical to perform the average numerically. However, at high enough frequencies where the length of the body is much greater than a wavelength, the energies from the gas and tissue will add independently:

$$
\left\langle\sigma_{\text {bs }}\right\rangle=\left(\sigma_{\text {bs }}\right)_{\text {bubble }}+\left\langle\sigma_{\text {bs }}\right\rangle_{\text {tissue }}, \quad k_{1} L \gg 1 .
$$

There are no brackets around $\left(\sigma_{\mathrm{bs}}\right)_{\text {bubble }}$ since the scattering by the bubble is independent of orientation.

\section{COMPARISON BETWEEN MODELS AND DATA}

The above theoretical models are now compared with laboratory scattering data from Stanton et al. (1997) involving single pings, ping-to-ping variability of the echoes from individual animals, and averages of the echoes.

\section{A. Single ping data}

There was reasonable success in using the models to qualitatively predict the various classes of patterns of measured target strength versus frequency (Fig. 2). 


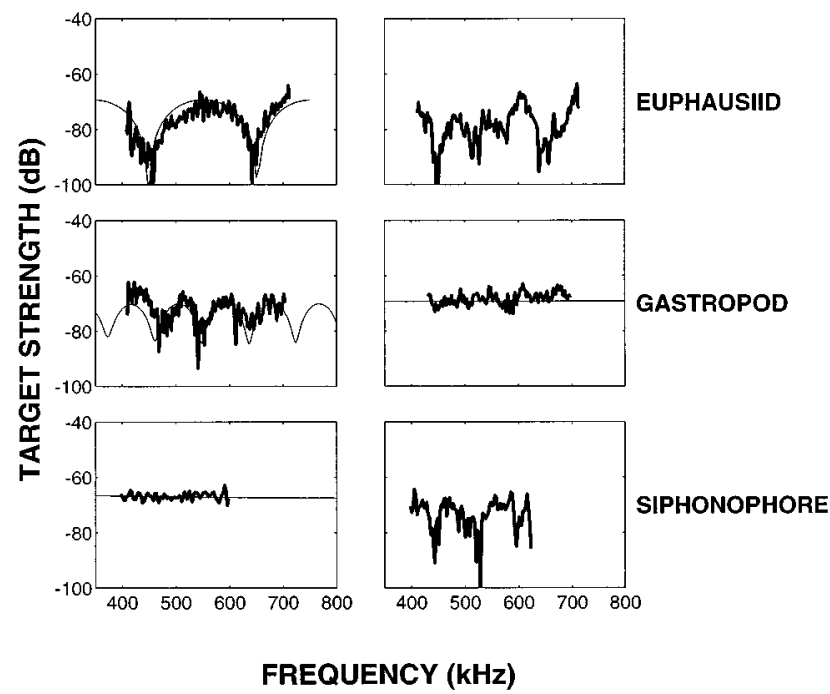

FIG. 2. Target strength versus frequency for individual echoes from a euphausiid, two different gastropods, and a siphonophore. Two-ray models are given in the (left) euphausiid and gastropod plots where the structure of the data is regular. A one-ray model is used for the nearly flat gastropod curve while the exact gas sphere model is used for the flat siphonophore curve. Thin curves are model predictions, thick curves are data. Description of the animals is given in Fig. 2 of Stanton et al. (1998). Simulation parameters are a combination of measured values, values published for similar animals or materials, semi-empirical model-based values, and values inferred from the data: euphausiid, left panel only: Eq. (15) used with $\theta=0,(g, h)$ $=(1.0357,1.0279)$ based on properties measured for Euphausia superba (Foote, 1990 and Foote et al., 1990), $a=1.9 \mathrm{~mm}$ (within range of measured values, used for best fit to structure of plot), $\rho_{c} / L=2.2$ (consistent with visual observations, used for best fit to overall levels of data), $L=29 \mathrm{~mm}$ (length, based on measurement, is "reduced" acoustic length since tail contributes so little to the scattering); gastropod, left panel: Eq. (31) used with $\mathscr{B}_{12}=0.84, a=\bar{a}=0.63 \mathrm{~mm}$ (this is the radius of a sphere whose volume is the same as the gastropod modeled as a 2 -mm-long by 1 -mm-wide prolate spheroid), $\sigma=.025 \bar{a}$ (inferred from data), $\beta_{L}=0.002 k_{1} a$ (the $k a$ functional dependence is based on the analytical Lamb-wave model and the coefficient 0.002 is based upon a fit to the data), $\alpha_{L}=8 k_{1} a$ (based on model), $\Phi_{L}=$ $-\pi / 2$ (inferred from data) for the subsonic wave, $\theta_{L}=\pi / 2$ (based on model), $c_{L} / c_{1}=k_{1} \bar{a} /\left(\alpha_{L}+0.5\right) \simeq 1 / 8$ (this value was predicted theoretically and observed), $F_{L}=1$ (inferred), $F_{\text {spec }}=0.71$ (the $\mathscr{R}_{12}=0.84$ is calculated for the coefficient expected for a semi-infinite planar half-space of calcite where the published values for calcite $g=2.646$ and $h=4.345$ (longitudinal waves), the inferred value of $F_{\text {spec }}=0.71$ in essence "corrects" that value down to 0.6 ), also, the series was truncated to include only the $m=0$ term in the Lamb wave series; gastropod, right panel: same as left panel except $a$ $=0.81 \mathrm{~mm}, F_{\text {spec }}=1$ and $F_{L}=0$ (the inferred value $F_{L}=0$ corresponds to total loss of Lamb wave due to change in orientation, change in $a$ from left panel corresponds to the incident wave "seeing" a different radius of curvature at front of gastropod due to change in orientation); siphonophore, left panel only: Eq. (34) using $a=0.35 \mathrm{~mm}$ (this is the radius of a sphere whose volume is the same as the elongated gas bubble modeled as a 1.3 -mm-long by 0.5 -mm-wide prolate spheroid $),(g, h)=(0.0012,0.22)$ which are published values for air at one atmosphere pressure.

Euphausiids. The regular pattern of TS versus frequency for the euphausiid can be described by the two-ray model (Fig. 2, left plot). The positions of the peaks and dips (or nulls) for this particular plot were satisfactorily described by the model indicating that the pattern was most likely due to constructive and destructive interference between rays from the front and back interfaces of the animal near broadside. It was not possible to make objective comparisons between the overall predicted echo levels and those observed as the pre- cise orientation and shape of the animal was not known. Here, the radius of curvature of the bent cylinder model was fixed at a reasonable value $\left(\rho_{c}=2.2 L\right)$ that also resulted in a best fit to the data and the orientation was assumed to be broadside for the modeling of the individual ping. Other echo data (not shown) from this animal showed the nulls changing position and spacing (typically smaller spacing) which indicates that changes in the orientation and shape will change the pattern. Adjustment of the relative phases of the rays in the two-ray model can account for this effect provided the pattern is periodic. For slight changes in phase, the adjustment corresponds to the (tapered) animal changing orientation so that a different part of its cross section with a different thickness is in the first Fresnel zone of the acoustic beam which dominates the scattering. Larger changes may correspond to the possibility of rays from other parts of the body contributing to the echo.

This work with a freshly caught euphausiid is consistent with the modeling performed on the decapod shrimp (Stanton et al., 1993a) where the two-ray model was first developed. In that work, the animals were oriented so that they were nearly broadside to the incident field. The regular patterns observed with the euphausiids reported in this more recent work also correspond to nearly broadside incidence. Visual observation of the orientation of the animal used in Fig. 2 was made periodically with a viewing window and indicated that the animal was generally near broadside throughout the experiment.

The irregular patterns in the euphausiid data observed in this study (Fig. 2, right plot) are similar to the ones observed in Stanton et al. (1994b) involving broadband insonification of an obliquely oriented decapod shrimp. It was determined in that analysis that the echo could be approximated from a statistical standpoint by as few as six randomized rays. The six rays were formulated with random phases and added together to form a random signal whose spectral characteristics are irregular. This type of signal is expected when the animal is either not at broadside incidence or near broadside but with an irregular shape. With this random set of rays, comparison of a single realization of the predicted echo with data is not particularly useful, hence a prediction is not shown. The statistical properties of the predictions and data are compared below.

Gastropods. The gastropod that exhibited a consistent periodic pattern of TS versus frequency was best modeled by a two-ray model where one ray was from the front interface of the body and the other ray was due to a single $(m=0)$ Lamb wave that traveled around the body once while experiencing roughness-induced attenuation (Fig. 2, left plot). The periodicity exhibited by the data is characteristic of the dominant subsonic zeroth order antisymmetric Lamb wave. Initial application of the model with no roughness resulted in a pattern (not shown) that had the same periodicity as the one measured, but with sharp peaks and nulls that did not resemble the data. Once roughness was incorporated, the $m$ $>0$ Lamb waves were attenuated to the point that they did not significantly contribute to the scattering. The result was two terms dominating the expression, the echo from the front interface and a (roughness-induced) attenuated Lamb wave. 
With roughness incorporated, the resultant pattern is more smoothly varying like the data and exhibits the same periodicity as that of the data. In the computations, the infinite Lamb wave series must be truncated at some point. We chose to truncate at the $m=0$ point (i.e., to include only the $m=0$ wave) which further improved the fit to the data.

In contrast to the above gastropod whose particular orientation was apparently conducive to the Lamb wave traveling readily around the body, another gastropod was oriented in such a way so that the Lamb waves were apparently blocked (Fig. 2, right plot). The resultant pattern is relatively flat and was modeled simply by eliminating the Lamb wave term in the model (i.e., by setting $F_{L}=0$ ). It is hypothesized in this case that the opening of the shell was facing in a direction that prevented the traveling of Lamb waves in paths along the body that would have eventually shed toward the receiving transducer.

A major challenge in modeling the scattering by the gastropods involved choice of material properties and associated Lamb wave coupling coefficients and speed. The animal bodies consist of aragonite. Since all critical material parameters (density, longitudinal sound speed, and shear sound speed) were not available for aragonite, we used published parameters associated with calcite, a very similar substance. The density and longitudinal speed were used directly for predicting the Rayleigh reflection coefficient. Given the difficulty of determining the Lamb wave parameters for a body of this complexity, we used certain values inferred from the data. We also compared numerical calculations of Lamb wave parameters based on backscattering by an idealized spherical shell with the inferred values to test the validity of the hypothesis that the observed subsonic waves are indeed the zeroth-order antisymmetric Lamb wave.

The shell of the gastropods used was approximately 5 $\mu \mathrm{m}$ thick according to our measurements of shell thickness of other similar-sized animals. We are uncertain as to the thickness of the shell within all parts of the body and assume that layering of the spiral shell will cause the thickness to vary within a given shell. Because of the variation in thickness, it is expected that there would be deviation between predictions of Lamb wave parameters based upon an idealized sphere and comparisons with the data.

Using the calcite material parameters, predictions of acoustic backscattering were made for individual water-filled spherical shells and for individual tissue-filled shells, each for a variety of shell thickness (the thickness was held fixed for a given simulation) (Kaduchak, 1997). The predictions were based upon the Sommerfeld-Watson transformation of the modal series solution. This transformation converts the modal series into a series of ray terms (specular ray, Lambwave ray, etc.). The tissue material properties were chosen to be the similar to those of weakly scattering fluidlike animals (density and sound speed were set equal to 1.1 times that of the surrounding water) for lack of available information.

The idealized shell calculations show that for a 5- $\mu \mathrm{m}$ thick shell and $k a$ in the range of 1-4, the speed of the zeroth-order antisymmetric Lamb wave was roughly $\frac{1}{8}$ that of the surrounding water. This value is the same as that which we observed in the measurements. For shells of $20-\mu \mathrm{m}$ thickness, the predicted value was about $\frac{1}{3}$ that of the surrounding water, which is much greater than what we observed. However, the predictions of $\beta_{L}$ produce values of about 0.005 for the $20-\mu \mathrm{m}$-thick shell for $k a=1$ and values very close to zero for higher $k a$ and/or thinner shells. The inferred value of $\beta_{L}$ (from the data) was about 0.002 once roughness was taken into account (if roughness was not taken into account, that inferred value would be smaller but not small enough to be consistent with a value predicted for 5 - $\mu \mathrm{m}$-thick idealized shells). Thus one inferred value $\left(\beta_{L}\right)$ is consistent with predictions from a shell thicker than that associated with the predictions that were consistent with the other inferred value $\left(c_{L}\right)$. Nonetheless, both inferred values involving data from the rough irregular animal shell of variable thickness were consistent with the range of predictions involving a range of (idealized) shell thickness close to that of the animal, which is perhaps the best one could expect given the differences between the animal shell and the idealized spherical shell. These inferred values therefore appear to be reasonably consistent with the hypothesis that the subsonic wave is the zeroth-order antisymmetric Lamb wave.

Given the facts that the shell thickness is nonuniform and that the observed subsonic wave had significant energy for values of $k a$ greater than unity (which implies a nonzero value of $\beta_{L}$ ), we used the values of the Lamb wave speed and coupling coefficient that were inferred from the data rather predicted from the idealized predictions.

Siphonophores. The nearly flat pattern exhibited in some echoes by the siphonophore could be modelled by the single bubble model (Fig. 2, left plot). The single bubble alone will give a flat spectrum in this frequency range regardless of angle of orientation. Alternatively, the flat pattern could be modeled by the more general bubble-plus-tissue model for realizations in which the tissue does not contribute significantly to the scattering. The irregular pattern (Fig. 2, right plot) must be predicted by the model which includes both the gas and tissue. Because of the stochastic nature of the scattering by the tissue, it is not useful to make direct comparisons between single realizations of the predicted and measured patterns. The statistics of the predicted and measured echoes can be compared and are done so in the next section.

\section{B. Orientation dependence of echoes}

Orientation of one of the euphausiids was digitized from the video camera data and compared with the echo levels that correspond to each video frame (Fig. 3). Scattering predictions based upon the DWBA model using observed orientation data were also compared with the observed scattering levels (Fig. 3).

The data illustrate that the echoes are generally higher for broadside incidence than for end-on. There was also significant variability from ping-to-ping in the echo level. Because of the variability and for direct comparisons, the scattering data, scattering predictions, and orientation data were averaged over a running seven-ping interval (before the averaging was performed the scattering predictions for a given ping were based on the observed instantaneous (i.e., not averaged) orientation angle). Also, the data and simulations represent an average over the $350-600-\mathrm{kHz}$ spectrum, 

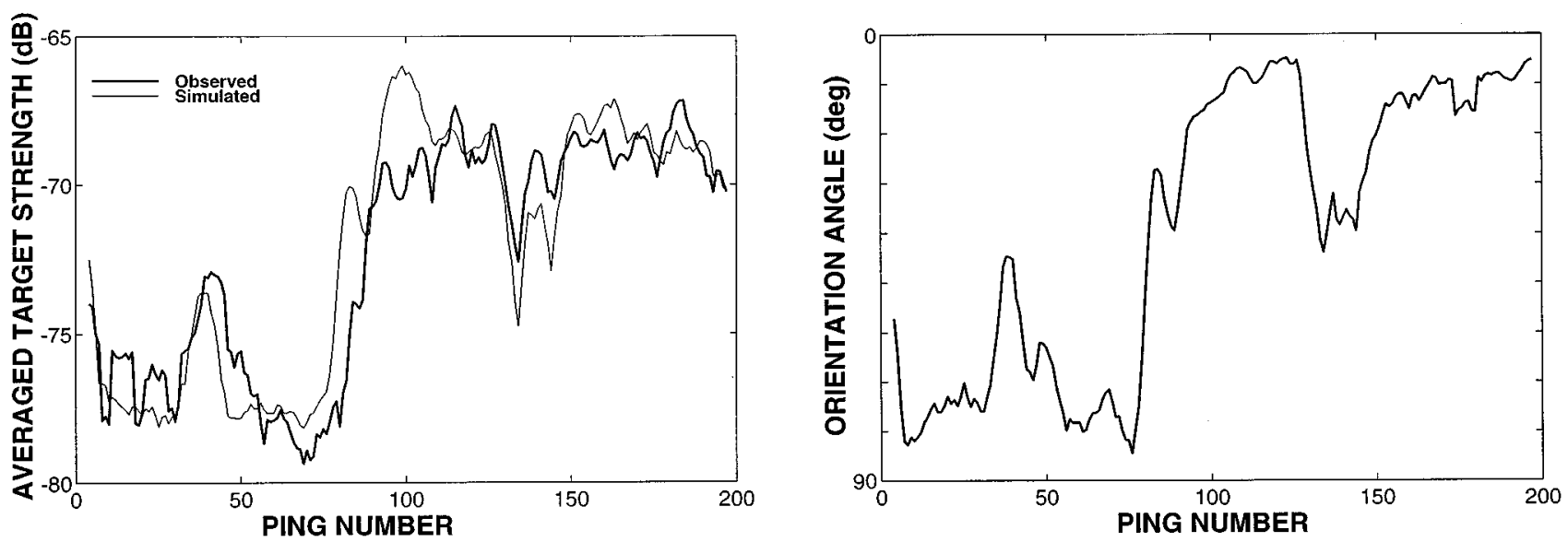

FIG. 3. Average target strength data from an individual 36-mm-long euphausiid and predictions from 200 consecutive acoustic pings as animal changes orientation [same animal as in Fig. 3 of Stanton et al. (1998)]. Left plot: Measured and simulated acoustic backscattering. The measured scattering has had the measured noise (equivalent TS $=-81.5 \mathrm{~dB}$ ) removed from it (removed on a linear scale first before logarithm is taken). The simulations use a rough tapered inhomogeneous cylinder model, based on the DWBA line integral given in Eq. (5). Right plot: measured orientation angle of euphausiid averaged over seven-ping running average for smoothing purposes. Scattering and orientation data collected simultaneously for each ping. Predictions use same (instantaneous, not averaged) orientation angle as measured for each corresponding ping although head-tail reversals are not distinguished for the near-broadsideincidence data. Pings 10-30 and 60-70 involved near head-on incidence and pings 100-120 and 150-200 involved near broadside incidence (where the head of the body was observed to be, on average, slightly farther from the transducers than the telson or "tail"). The measured and simulated backscattering levels were averaged over a seven-ping window (a simple uniformly weighted seven-ping average for the data and a 30-realization Gaussian-distributed average based on the mean and standard deviation of angles over the seven pings used for the simulations). The backscatter data and simulations were averaged over the $350-600-\mathrm{kHz}$ band of frequencies (all averaging, over pings and frequencies, involved averages of backscattering cross-section before the logarithm was taken). The measured noise and unwanted reverberation was reduced through first time-gating the compressed pulse output (Chu and Stanton, submitted) and had (after processing) an equivalent average target strength of $-81.5 \mathrm{~dB}$ resulting in the worst case SNR of about $2.5 \mathrm{~dB}$ near ping number 70 (near end-on incidence), but typically about $4 \mathrm{~dB}$ and better for other near end-on incidence values and better than about $11 \mathrm{~dB}$ for near broadside incidence. Simulation parameters: $L=30.5 \mathrm{~mm}$ (this measured quantity is the reduced or acoustic length that corresponds to the fact that the telson or "tail" of the euphausiid does not contribute substantially to the scattering), mean cylindrical radius $\bar{a}=1.9 \mathrm{~mm}$ (inferred from measurement of length), $\rho_{c} / L=0.8$ (this is an intermediate value within the range of values of the ratio as measured from the video images), and the mean values $\bar{g}=\bar{h}=1.054$. These values of $\bar{g}$ and $\bar{h}$ were adjusted for a better fit to the data and are higher than the values in other simulations in this paper. The value of $\rho_{c} / L$, which is based on measurements, is lower than that used in other simulations in this paper. The higher values of $g$ and $h$, in essence, offset the decrease in scattering levels due to the decrease in $\rho_{c} / L$, making this combination of $g, h$, and $\rho_{c} / L$ somewhat compatible with the combinations used in other simulations. The values of $g$ and $h$ varied independently and randomly (Gaussian distributed) along the length of the body with a standard deviation of 0.005 over six segments of equal length. The value of radius varied independently and randomly (i.e., uncorrelated adjacent values, each following a Gaussian distribution with a standard deviation of $10 \%$ of the expected value) over 200 segments of equal length.

which corresponds to the spectrum of useful energy of the transducers. Because of the low signal-to-noise ratio of some of the echoes, the average measured noise level was subracted from the measured (noisy) echo levels which resulted in a better representation of actual measured target strength.

The scattering modeling for angles of orientation near broadside was relatively straightforward as the levels were predictable using smooth homogeneous bent tapered cylinders. However, for angles near end-on, it was a significant challenge to predict the echo levels. This fact, coupled with the facts that (a) there was variability from ping to ping in the echoes as the animal changed orientation slowly and (b) the statistics of end-on echoes were consistent with echoes due to many parts of the bodies (Stanton et al., 1994b; Fig. 4 of this paper), required a more complex shape and material profile to be used. As shown in Stanton (1992) and Stanton and Chu (1992), roughness of bounded bodies can cause significant variability in echoes and has been used to explain ping-to-ping variability of tethered animals (Wiebe et al., 1990). Roughness elements of a target can be treated as individual scatterers. Once the target is at an oblique angle to the sonar transceiver and the main returns are not coming from the front and back interface, then these individual elements play a significant role in the scattering. It is quite possible that the roughness elements are the source of the multiple ray echoes observed near end-on. Adding to the complexity of the problem is the possibility that the material property of the animals is not uniform throughout the body. The inhomogeneities of the material properties could also give rise to scattering levels near end-on.

Predictions using a smooth homogeneous cylinder model underestimated the scattering levels near end-on incidence. For the reasons given above, we used a more complex description of the animal morphology in order to describe the near end-on scattering levels. We used an elongated teardrop tapering function much like that illustrated in Fig. 1 of Stanton (1992) and incorporated both roughness and inhomogeneities of material properties of the body. Given the lack of knowledge of the precise roughness and material profile, we arbitrarily assigned $10 \%$ random variability to the cross-sectional radius along the length of the body and $0.5 \%$ random variability to the mass density and sound speed along the length of the body [the $0.5 \%$ variability corresponds to about $10 \%$ of the difference between the (weakly scattering) body material properties and that of the surrounding water].

The actual roughness profile of the animals has both a small smoothly varying component (dorsal side) and large 
rapidly varying component (ventral side which contains legs). For simplicity, an intermediate (10\% of radius) rapidly varying profile was used which involved statistically independent rapidly varying values of cross-sectional radius. The material property profile consisted of six statistically independent sections to represent the fact that there are a small number of sections of the body of a euphausiid.

With these added complexities, we made predictions of acoustic scattering over the entire range of orientation angles (Fig. 3). The predictions of backscattering with the rough inhomogeneous bent tapered cylinder generally follow the pattern of the measured levels over the full range of orientation angles. Most importantly, the near-end-on levels could be predicted by taking into account the added complexities of the model (the predictions near broadside were not significantly affected by taking into account the added complexities). Although the modeling of the complexities was far from ideal in this particular study, it is clear that the finescale complexities of the animal body must be included in order to predict near-end-on backscattering.

\section{Statistics of echoes}

The statistics of some sets of echoes are analyzed for one euphausiid and one siphonophore. The animals were free to change orientation throughout the measurements and many orientations and shapes were realized. However, because of the small size of the gastropods and nature of the tethering process, their movement was highly constrained and a statistical analysis is less meaningful and not included.

\section{Euphausiids}

The statistical behavior of the echo envelopes of the scattered signal from the euphausiid was analyzed with both the ray approach (Fig. 4) as well as with the DWBA approach (Fig. 5). The ray approach helps provide physical insight into the scattering process, although the results for this type of approach tend to be qualitative. The DWBAbased method, while more complex than the ray approach, is also potentially more predictive. Given the significant difference in the variability of the echoes between the cases involving broadside and end-on incidence, the analysis treats each of those cases. We perform this statistical analysis on the data set presented in Fig. 3 in which the video data of the animal were used to measure animal orientation for each of the echoes measured. Only the $560-\mathrm{kHz}$ components of the broadband echoes were examined in this statistical study (data and simulations).

Ray approach. Depending upon the orientation information as well as pattern of target strength versus frequency, either the two-ray model or the six-ray model was used in the ray description (Fig. 4). The two-ray model is used in the case when the animal was generally near broadside during the measurements, which resulted in a regular pattern for about $40 \%$ of the echoes. The six-ray model is used in the case when the animal was generally near end-on (head-on in this case), which resulted in an irregular pattern for about $90 \%$ of the echoes. In the two-ray model, variations in orientation angle and shape are taken into account by randomly and uniformly varying the cylindrical radius of the animal
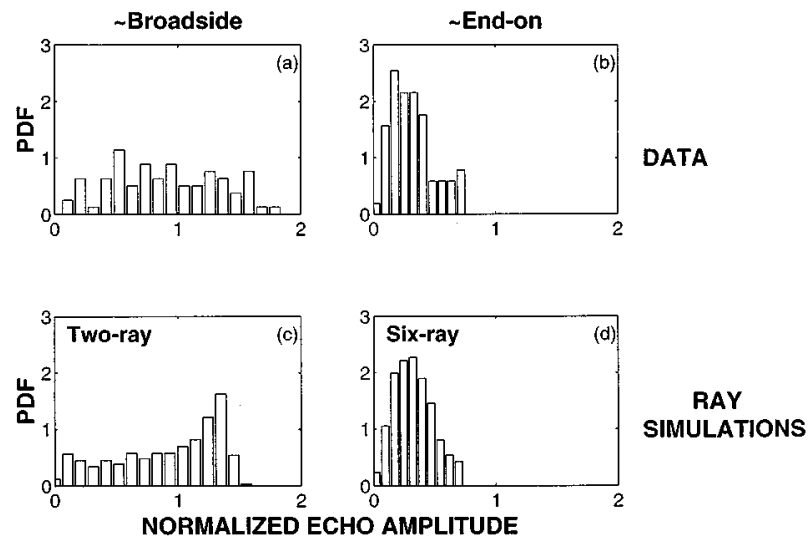

FIG. 4. Echo envelope histograms of echoes from an individual 36-mmlong euphausiid from sets of pings in which the animal is either generally broadside or end-on to the incident acoustic wave (same animal as in Fig. 3 of Stanton et al. (1998) and using subset of data presented in Fig. 3 of this paper). Acoustic frequency is the $560-\mathrm{kHz}$ component of the broadband echo. Two-ray $(N=2)$ and six-ray $(N=6)$ models are used in those cases, respectively, using Eq. (12). All $b_{j}$ for a given simulation [plots (c) and (d)] are of the same value. The horizontal scale of each of the four plots is normalized by the rms amplitude of the values plotted in plot (a). The end-on and broadside pings were selected from a fraction of pings within the range of ping numbers $5-70$ and $100-200$, respectively. The mean (near) end-on angle is measured to be $14.1^{\circ}$ off head-on incidence $\left(\right.$ s.d. $\left.=9.9^{\circ}\right)$ and the mean (near) broadside angle is measured to be $10.1^{\circ}$ off broadside incidence (dorsal aspect) $\left(\mathrm{s} . \mathrm{d} .=5.1^{\circ}\right)$ with the head of the body observed to be slightly farther, on average, by about $10^{\circ}$ from the transducers than the telson or "tail." Certain pings within those ranges were excluded; for example, pings near pings 40 and 140 were excluded where intermediate angles were observed.

over a range of values within $\pm 30 \%$ of the average cylindrical radius (this distribution coincidentally corresponds to a uniform distribution in phase difference between the two rays over the $0-2 \pi$ range at $560 \mathrm{kHz}$ ). This randomization
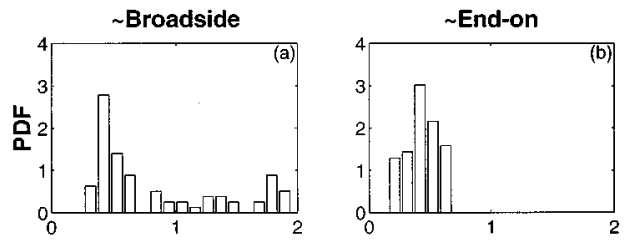

DWBA ROUGH/INHOMO
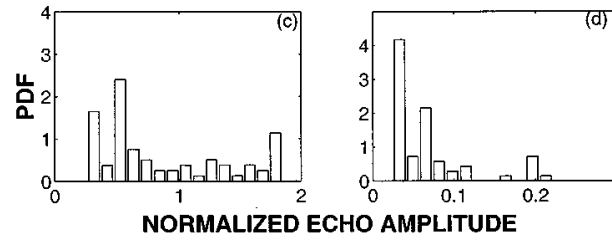

DWBA SMOOTH/HOMO

FIG. 5. Echo envelope histograms of simulated echoes from an individual 36-mm-long euphausiid using two DWBA-based models. The predictions were based on the simulations performed in Fig. 3 using selected pings for broadside and end-on incidence. The rough and inhomogeneous model used the $560-\mathrm{kHz}$ component of the Fig. 3 simulations directly using the same broadside and end-on pings that were used in the data plots as in Fig. 4. The smooth and homogeneous model used the $560 \mathrm{-kHz}$ component of simulations that were similar to those performed for Fig. 3, using the same broadside and end-on pings that were used in the data plots in Fig. 4, but with no variability in radius (except for the taper) or in material properties. The simulations for these four plots excluded noise. 
takes into account the fact that the animal has a crosssectional radius that varies along the length of the body. When it changes orientation, a different part of the body (with a different radius) will make up the first Fresnel zone of the scattering and dominate the scattering.

Both measured and predicted echo amplitude histograms show marked differences between the two cases of animal orientation. The histograms for the data involving near broadside incidence show a more-or-less featureless pattern. Those histograms can be explained, in part, by first examining the model shown in the left plot of target strength versus frequency in Fig. 2. For a two-ray model, one expects there to be echo levels spanning the full range of values from 0 (total destructive interference) to twice the level of one ray (constructive interference) with more values occurring near the maximum value. The corresponding histogram of echo envelope values based on the two-ray simulations takes on a set of nearly uniformly distributed values up to the maximum echo value which has a peaked histogram value. The data histogram is similar to the simulated pattern with differences at the high echo levels - the data do not show a peak in the histogram near the maximum echo value.

In contrast, the histograms for the case of end-on incidence show a Rayleigh-like PDF with a distinct peak. The data for end-on incidence here have a lower SNR than in the broadside case which adds a Rayleigh-distributed component to the echo. However, the (Rayleigh-like) variability of these end-on incidence echoes are consistent with the variability at end-on incidence observed in an earlier experiment with a similar elongated animal (decapod shrimp), but with a higher SNR (Stanton et al., 1994b). In order for the envelope of the scattered signal to be Rayleigh distributed, the scattering must be random and diffuse, which implies that there are echoes coming from many parts of the body that contribute significantly to the total echo.

With both classes of animal orientation, the ray simulations predicted the general nature of the histograms involving the euphausiids. In addition to the six-ray model also being consistent with the end-on decapod shrimp data and associated ray modeling presented in Stanton et al. (1994b), the two-ray model is also consistent with the broadside decapod shrimp data and ray modeling presented in Stanton et al. (1993a, 1994b) confirming the earlier results for animals of this body form.

DWBA approach. In addition to the more qualitative ray-based approach, we also investigated the statistical nature of the echo with the more predictive DWBA method (Fig. 5). Both a smooth homogeneous asymmetrically tapered bent cylinder as well as a rough inhomogeneous asymmetrically tapered bent cylinder were used. The simulation parameters for the latter case are identical to those used in the study of backscatter versus angle described in Fig. 3 while using the orientations used in Fig. 4 for direct comparison. The parameters for the smooth-homogeneous-model simulations were identical to those of the roughinhomogeneous-model case in every respect except for the fact that the mean values of the cylindrical radii and mass density and sound speed contrasts from the rough inhomogeneous case were used rather than the stochastic values (the radius still varied according to the same taper function).

The DWBA-based simulations show that the variability of the echoes for angles of incidence near broadside for the rough inhomogeneous case is broadly similar to that of the smooth homogeneous case (left plots in Fig. 5). The echo envelope PDFs based on both sets of DWBA simulations for broadside incidence span a relatively wide range of echo values like the echo data PDF presented in the top left plot of Fig. 4. However, the data PDF has much less structure than either of the sets of DWBA-based simulations.

The greatest differences between the DWBA-based approaches involved end-on incidence. The mean value of the predictions for the smooth, homogeneous case was significantly lower than the corresponding value for the rough, inhomogeneous case (right plots in Fig. 5). The difference in mean level is consistent with the differences observed between the two different models averaged over the band of frequencies that were discussed above. Furthermore, the shapes of the two histograms in the end-on case are also different from each other. The shape of the PDF of the echo envelope data (top right plot in Fig. 4) is closer to the shape predicted by the rough inhomogeneous model than that predicted using the smooth homogeneous model, although there are some distinct differences.

In conclusion, predictions of the echo envelope statistics appear to be relatively insensitive to the complexity of the DWBA model (rough inhomogeneous versus smooth homogeneous) for the distribution of angles of incidence near broadside. The greatest sensitivity involved the case where the distribution of angles was near end-on incidence. Here, the rough inhomogeneous model explained the overall (mean) levels of the data much better than for the predictions involving the smooth homogeneous model and it explained the shape of the PDF data somewhat better. The improvements are due to the fact that there are elements both within the body interior and on the surface that can contribute to the scattering. These elements are insignificant near broadside incidence as the echoes from the front and back interface tend to dominate the scattering. However, off normal incidence, the two rays do not contribute significantly to the scattering and effects due to these other sources of scattering dominate. The fact that these multiple elements dominate near end-on is consistent with the fact that a six-ray model is required to describe the scattering in that region (Fig. 4).

\section{Siphonophores}

In the case where the siphonophore was studied both whole and without its pneumatophore, the measured echo envelope PDFs were markedly different (Stanton et al., 1997, and Fig. 6 of this paper). When comparing the predictions with the data, a model including both the gas contribution (one ray of constant phase) and tissue contribution (six randomized rays) was used to describe the whole animal while the six-ray model alone was used to describe the scattering by the animal without its pneumatophore. The phase of the gas ray was held constant while the phase of each tissue ray was randomized uniformly over the range 0 to $2 \pi$. The use of six rays was chosen to describe the scattering by the tissue because of the generally random shape and ori- 

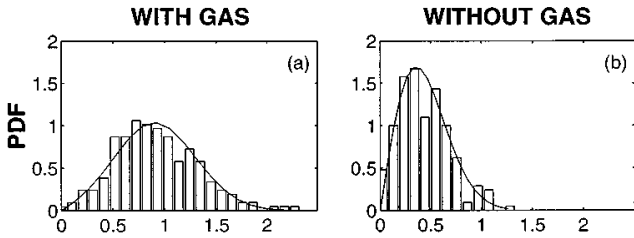

DATA
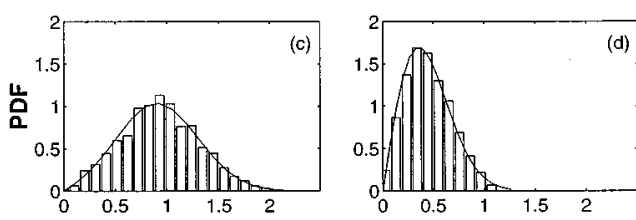

SIMULATIONS

NORMALIZED ECHO AMPLITUDE

FIG. 6. Statistical study of echoes from an individual siphonophore both whole and with pneumatophore removed. Measured data (histograms in upper plots) are compared with simulation histograms (lower plots). Rice PDF curves are superimposed upon all histograms. Data taken directly from Fig. 6 of Stanton et al. (1998). Acoustic frequency is the 560-kHz component of the broadband echo. The horizontal scale from each plot is normalized by the rms value of the values plotted in (a). Rice PDF shape parameter: using a $\left(\gamma_{\mathrm{RICE}}\right)$ notation similar to that $(\gamma)$ of Stanton and Chu (1992), $\gamma_{\mathrm{RICE}}=2$ for both plots in left column and $\gamma_{\mathrm{RICE}}=0$ for both plots in right column where $\gamma_{\text {RICE }}$ is the ratio of coherent to incoherent energy of the signal [this $\gamma_{\mathrm{RICE}}$ and the $\gamma$ in Stanton and Chu (1992) are not to be confused with the other $\gamma$ given explicitly in the text of this paper]. For $\gamma_{\mathrm{RICE}}=0$, the Rice PDF reduces to the Rayleigh PDF. The simulations in the left plot used Eq. (12) inserted into each of the two terms on the right-hand side of Eq. (33). For the bubble, $N=1$ and $\epsilon_{j}=0$, while for the tissue $2 k_{1} \epsilon_{j}$ was randomized in the range $0-2 \pi$ and $N=6$ (six random rays from the tissue). The energy of each tissue ray was set equal to 0.083 of the energy from the gas ray to be consistent with the observed $\gamma_{\mathrm{RICE}}=2$. The simulations in the right plot use the same ray summation as in the left plot, but now excluding the gas term.

entation of the body which would tend to produce diffuse scattering at these high frequencies. As described above, six rays is a reasonable approximation for describing the statistics of a diffuse scatterer.

In order to further understand the statistics of the echoes and to determine the relative value of the rays, the Rice PDF is used in this analysis. The Rice PDF was originally derived in electrical signal theory to describe the envelope of a sine wave with added noise (Rice, 1954). The PDF has since been applied to various scattering problems where there may exist a consistent echo with a highly variable one superimposed (Clay and Heist, 1984; Stanton, 1984; Stanton and Chu, 1992). In the case of the siphonophore, the consistent echo would correspond to the scattering by the gas and the variable echo would correspond to the scattering by the tissue. Fits of the Rice PDF to the siphonophore data result in values of the shape parameter $\gamma_{\text {RICE }}$ to be equal to 2 and 0 for the whole animal and animal-less-gas data, respectively (Fig. $6)$. In this context $\gamma_{\text {RICE }}$ is defined as the ratio of coherent scattered energy to incoherent scattered energy. The value of $\gamma_{\mathrm{RICE}}=2$ for the whole animal indicates that the incoherent energy is $\frac{1}{3}$ of the total energy. The observed decrease of about $5 \mathrm{~dB}$ in echo energy when the gas inclusion was cut off is consistent with this fraction $(10 \log (1 / 3) \simeq-4.8 \mathrm{~dB})$. The value of $\gamma_{\mathrm{RICE}}=0$ for the tissue-only case corresponds to the limiting case of the Rice PDF when it becomes a Rayleigh PDF (i.e., diffuse random-phase scattering with no coherent component), while the $\gamma_{\text {Rice }}=2$ case corresponds to a more Gaussian-like PDF. For this latter, whole animal, case the relative strengths of the rays in the ray modeling can be estimated. The value $\gamma_{\mathrm{RICE}}=2$ implies that the sum of the energies of the six (tissue) rays is 0.5 times that of the simulated gas echo. By arbitrarily assigning equal strength to each tissue ray, then the energy of each tissue ray would be 0.083 times that of the energy of the gas ray.

Using the relative values for the ray strengths as estimated above, there is reasonable qualitative agreement between the ray simulations of echo envelope histograms and data (Fig. 6). Data involving the whole animal and corresponding simulations show the distribution of the echo envelope to be Gaussian-like. Data and simulations involving the animal without the pneumatophore show the distributions to be Rayleigh-like. All histograms are consistent with the Ricean PDF for different $\gamma_{\text {RICE. }}$ As discussed in Stanton et al. (1997), this variability in shape of echo histogram for the animal with and without the gas is consistent with the hypothesis that the gas produces a strong consistent return. The tissue at these high acoustic frequencies produces a highly variable return, indicating that there are interfering echoes coming from various parts of the tissue.

\section{Averaged echoes}

The backscattering cross sections from individual animals were averaged over a large number of pings and compared with the appropriate models on a decibel scale (Fig. 7). Data used are from a larger range of acoustic frequencies than with the single ping analyses.

The averaging has the general effect of smoothing out most of the structure of the scattering. Some structure remains in the euphausiid data where there is a dip and peak at 500 and $600 \mathrm{kHz}$, respectively. The structure in the siphonophore data quite expectedly disappeared as the echo from the gas should be relatively strong, featureless, and stable while the structure from the echo due to the tissue should be random with no consistent structure. The structure in the gastropod data mostly disappeared, possibly due to the variability in path length of the Lamb wave (and hence variability in structure) as the animal changed orientation.

Two models for the euphausiids were used in the predictions-both the more precise DWBA model numerically averaged over orientation and size and the two-raybased model analytically averaged over orientation and size. The size averages correspond to the fact that the animal is tapered and the part of the cross section that dominates the scattering depends upon orientation angle. Consequently, the section dominating the scattering for a given ping varies in diameter from ping to ping which results in an effective change in animal size. The average over a small range of length is equivalent to an average over a small range of radius in this case. The rough elastic shell ray model, numerically averaged over size, was used for the gastropods. The sum of the backscattering cross section using the exact modal series solution for a gas bubble and the cross section based on the analytically averaged bent fluid cylinder (ray) model for the tissue was used to model the scattering by the 

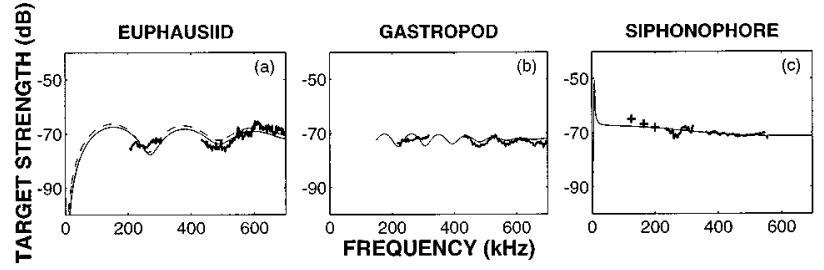

FIG. 7. Target strength versus frequency averaged over many echoes from euphausiid, gastropod, and siphonophore (cross section averaged before logarithm was taken). Both two-ray (dashed) and DWBA (solid) fluid cylinder models given in euphausiid plot, two-ray rough elastic shell model used for gastropod plot, and hybrid model of fluid bent cylinder containing gas sphere used for siphonophore plot. Thin smoothly varying curves (dashed and solid) are predictions, thick irregular curves are data from broadband transducers, and " + " are data from single frequency transducers. Predictions using the euphausiid curves with similar parameters that successfully predicted scattering levels down into the Rayleigh scattering region at $50 \mathrm{kHz}$ with decapod shrimp (Stanton et al., 1993b). Predictions similar to the siphonophore curve (but with two gas spheres to model split gas inclusions) follow the increase in levels near the resonance region as far down as $50 \mathrm{kHz}$ (Fig. A4 of this paper). Plot parameters: (a) euphausiid: same (individual) animal as in Fig. 2 of this paper, data averaged over first 50 pings of data from each transducer; Eq. (18) used for (analytically averaged) ray model using the same $(g, h)$ simulation parameters as in Fig. 2 and $L=29$ (measured reduced length), $a=1.75 \mathrm{~mm}$ (average value of measured radius at mid point in body, averaged between widthwise and depthwise directions), $s=0.08$ (estimated), $s_{\theta}=10^{\circ}$ (inferred), and the parameters $\left(C_{B}, T_{B}, \alpha_{B}\right)=(1.2,1,0.8)$ were previously determined through comparisons with DWBA predictions (Stanton et al., 1993b); an equation equivalent to Eq. (6) (but written in Cartesian coordinates) is used in (smooth, homogeneous) DWBA modeling $\left[\left|f_{\mathrm{bs}}\right|^{2}\right.$ in Eq. (6) was numerically averaged using above parameters (where appropriate) plus $\rho_{c} / L=3$ and a tapering function $a=a_{0} \sqrt{1-(z /(L / 2))^{10}}$ is used where $a_{0}$ is the radius in the middle of the body and $z$ is the position along the axis of the body relative to the $(z=0)$ mid-point of the body]. (b) Gastropod: averaged data from 50 pings each from eight individual (one at a time) Limacina retroversa. All animals were in the range $1.8-2.0 \mathrm{~mm}$ long. Numerical average of square of magnitude of Eq. (31) with the same parameters as given in Fig. 2 caption except for the inferred values $\Phi_{L}=\pi, F_{L}=1$, and $\sigma=0.06 \bar{a}$. The average spanned $\bar{a} \pm 3$ s.d. where s.d. $=0.1 \bar{a}$ (it is expected in this case for certain parameters with the average echo model to be different than those of the single echo model because for one realization, the ping will only "see" the front interface from one angle which has a fixed radius of curvature and related Lamb waves will only experience a certain range of radii whereas averaging over orientation involves the full range of values. (c) Siphonophore: one individual Nanomia Cara $26 \mathrm{~mm}$ long with only one gas inclusion $1.5 \mathrm{~mm}$ long by $1 \mathrm{~mm}$ wide [same siphonophore as used in Fig. 7 of Stanton et al. (1997)]. Here, 200 pings per frequency were averaged except for the $120-\mathrm{kHz}$ measurements where only 10 pings were averaged. Equation (37) was used with modal series solution [Eq. (34)] used for bubble component and averaged two-ray model [Eq. (18)] for tissue component. Model parameters are, gas: $a_{\text {sphere }}=0.425 \mathrm{~mm}$ based on measurement of bulbus side of gas inclusion facing the transducers, $(g, h)=(0.0012,0.22)$ based on published values for air at one atmosphere pressure. Tissue: $a_{\text {cyl }}=0.6 \mathrm{~mm}$ and $L=22 \mathrm{~mm}$ (both based on direct measurements of tissue section of body), $g=h=1.02$ (chosen to be consistent with those parameters of other weakly scattering bodies), $\left(C_{B}, T_{B}, \alpha_{B}\right)=(1.2,1,0.8)$ which were previously determined through comparisons with DWBA predictions (Stanton et al., 1993b), $s_{\theta}=0.15 \mathrm{rad}$ (inferred), and $s=1.0$ (this high length variability corresponds equivalently to the high variability in effective crosssectional radius of the body, $a_{\text {cyl }}$ is considered to be an effective radius because of the complex structure of the tissue section). The inclusion of the tissue scattering term adjusted the predictions at the highest frequencies upward by about $1.5 \mathrm{~dB}$, making the predictions more in line with the data.

(whole) siphonophore. The data and model for the siphonophore both indicate a rise in the levels for lower frequencies as the gas approaches resonance. The model predicts a resonance for this animal at about $4.6 \mathrm{kHz}$.

\section{SUMMARY AND CONCLUSIONS}

The scattering properties of zooplankton from various gross anatomical groups have been shown to be strongly dependent upon the material properties of the animals. The fluidlike euphausiids, hard-shelled gastropods, and gasbearing siphonophores all have distinctly different acoustic signatures. Mathematical models were used to relate the signatures to the basic physics of the scattering process.

The plots of (single ping) target strength versus frequency for individual euphausiids showed significant structure, regardless of orientation of the animals. The structure is sometimes regular for angles of orientation near broadside incidence, while it is random for all other angles. The type of structure for the gastropods varied dramatically with angle of orientation. For some angles, the structure was quite regular with pronounced peaks and nulls, while at others the pattern was more random. For some sets of angles, the pattern was nearly flat with random fluctuations of minimal amplitude. The siphonophores produced patterns that were sometimes nearly flat and sometimes irregular. Once the data were averaged over a set of pings, the structure of the target strength patterns tended to wash away. In some cases, there was some structure that would remain. For example, there is structure in the upper frequency range of the data for both the euphausiid and gastropod data in Fig. 7. However, the pattern of the residual structure varies with ping set. Other euphausiid data (not shown) have a different pattern of residual structure while other averaged gastropod data (Fig. 4 of Stanton et al., 1998) show no structure. No doubt, the orientation distribution of the animals for each ping set greatly affects the structure of the averaged data.

The greatest challenge in the modeling involved identifying the dominant scattering mechanisms and formulating reliable approximate formulas. Up to several models per animal were derived, depending upon the application and approximation used. The structure of both the target strength versus frequency data as well as compressed pulse output for single echoes provided most of the basis for the model development. The two-ray model (one ray from the front interface and one from the back) for the euphausiid seems to work well for orientations near broadside incidence and for averages over angle of orientation while more rays (corresponding to other parts of the body) are required for single ping data far off broadside. A predictive model incorporating roughness and material property inhomogeneities was also shown to describe scattering off broadside. A two-ray model is also required to model the gastropod in the geometric scattering region where one of the rays is from the front interface and the other is due to excitation of a Lamb wave on the shell. The siphonophore was characterized by the sum of an exact single bubble solution which was used to describe the scattering by its gas inclusion plus a model (DWBA or ray) for the tissue.

In conclusion, as a result of conducting controlled laboratory measurements, the fundamental scattering properties of zooplankton from several gross anatomical groups have been determined. This served as the basis for developing approximate acoustic scattering models which are predictive in nature. That is, they are written in a general enough form 
so that they can make predictions beyond the existing set of data and be used to describe scattering by other species within those groups. The models are limited, in part, by the amount of available scattering data. For example, direct measurements of the material properties of the animals need to be made to reduce the number of empirical parameters in the modeling. Certainly, more controlled experiments and sophisticated models will help improve the accuracy and range of usefulness of the existing models.

\section{ACKNOWLEDGMENTS}

The authors are grateful to Phil Marston of the Washington State University, Pullman, WA, for his advice on sound scattering by shelled bodies, Greg Kaduchak of the Applied Research Laboratory, University of Texas, Austin, TX, for his advice and numerical simulations determining Lamb wave parameters for fluid-filled spherical calcite shells over a wide range of conditions, and Shirley (Bowman) Barkley of the Woods Hole Oceanographic Institution, Woods Hole, MA, for preparing the manuscript to this article. This work was supported by the National Science Foundation Grant No. OCE-9201264, the U.S. Office of Naval Research Grant Nos. N00014-89-J-1729, N00014-95-1-0287, and N00014-94-1-0452, and the MIT/WHOI Joint Graduate Education Program. This is contribution number 9198 for the Woods Hole Oceanographic Institution.

\section{APPENDIX. EFFECTS OF FRAGMENTATION OF GAS INCLUSION OF SIPHONOPHORE ON ACOUSTIC PROPERTIES}

One challenge in the experiment involved maintaining the physical integrity of the siphonophores so that their morphology during the acoustic experiment represented its morphology in its natural environment. Some of the siphonophores were observed to have contained several gas inclusions. It is believed that during the netting procedure in which the animals were caught by the net at deep depths and raised to the surface, the gas inclusion of the siphonophore split into an array of smaller bubbles. The resultant scattering properties were dramatically different than that of an animal with a single inclusion. In this Appendix, the array of smaller inclusions is modeled to a first approximation as a linear array of $N$ equally spaced bubbles (Fig. A1). Scattering predictions based on this model are compared with data involving a siphonophore whose gas remained fragmented during the acoustic experiment.

The backscattering from a linear array of $N$ equally spaced bubbles at an arbitrary angle of orientation $\theta$ is written in terms of the backscattering amplitude $f_{1}$ of a single bubble as

$$
f_{N}=f_{1} \sum_{j=1}^{N} e^{-i 2(j-1) k_{1} D \sin \theta},
$$

where $D$ is the center-to-center separation between the bubbles. This equation was adapted from Eq. (12) by setting $b_{j}=f_{1}$ and $\epsilon_{j}=-(j-1) D \sin \theta$. The relative phase of each scatterer due to its respective position in the array is indicated in the summand. This equation is approximate as it assumes first-order scattering only. That is, any multiple scattering between the bubbles is ignored, as are any shadowing effects.

The average scattering cross section can be derived by first calculating the square of the magnitude of the above expression, then averaging over all angles of orientation assuming that the angles are uniformly distributed over the range $0-2 \pi$. The resultant expression can be written in compact form as

$$
\left\langle\left(\sigma_{\mathrm{bs}}\right)_{N}\right\rangle=\left(\sigma_{\mathrm{bs}}\right)_{1} A,
$$

where

$$
\begin{aligned}
A & =\sum_{j=1}^{N} \sum_{j^{\prime}=1}^{N} J_{0}\left(2\left(j^{\prime}-j\right) k_{1} D\right) \\
& =N+\sum_{j=1}^{N} \sum_{j^{\prime}=1}^{N} J_{0}\left(2\left(j^{\prime}-j\right) k_{1} D\right), \quad j \neq j^{\prime} .
\end{aligned}
$$

Here, $\left(\sigma_{\mathrm{bs}}\right)_{1}=\left|f_{1}\right|^{2}$ and $J_{0}$ is the zeroth-order Bessel function. The latter expression for $A$ is given as it helps show its limiting values:

$$
A \rightarrow \begin{cases}N^{2}, & 2(N-1) k_{1} D \ll 1, \\ N, & 2 k_{1} D \gg 1\end{cases}
$$

For very high frequencies where $2 k_{1} D \gg 1$, the echoes from the individual bubbles add incoherently (i.e., the phases of the echoes from the individual bubbles are randomly distributed over the range $0-2 \pi)$ and the average backscattering cross section is equal to $N$ times the cross section $\left(\sigma_{\mathrm{bs}}\right)_{1}$ of an individual bubble. For very low frequencies where $2(N$ -1) $k_{1} D \ll 1$, the echoes from the individual bubbles add coherently (i.e., the phases of these echoes are essentially the same) and the average cross section is equal to $N^{2}$ times the cross section of an individual bubble. For intermediate values of frequencies, $A$ will take on a much more complicated dependence upon the parameters of the array as given in the general equation above.

For one or two bubbles, $A$ reduces to simple forms:

$$
\begin{aligned}
& A=1, \quad N=1, \\
& A=2\left(1+J_{0}\left(2 k_{1} D\right)\right), \quad N=2,
\end{aligned}
$$

where for one bubble, $A$ is simply equal to unity (i.e., the average cross section is equal to the cross section of a single bubble, as expected), and for two bubbles, $A$ is equal to the sum of two terms. For low frequencies, the Bessel function term in the two-bubble expression becomes equal to unity making $A=4$ (i.e., coherent addition), while at high frequencies, the Bessel function approaches zero and $A=2$ (i.e., incoherent addition).

The scattering by the siphonophores whose gas inclusion remained fragmented was characterized by a target strength versus frequency pattern that contains significant structure (Fig. A2). The pattern was irregular and consisted of one or more peaks and dips (or nulls) that varied in position and level from ping to ping. Occasionally, the animals that produced this type of pattern would produce a flat pattern. It is hypothesized that the irregular pattern is due, in part, to interference between the echoes from the different gas inclu- 


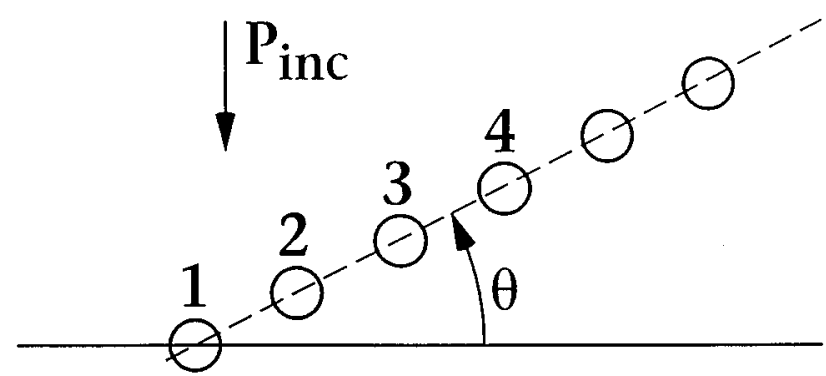

FIG. A1. Simplified scattering geometry for array of gas inclusions in siphonophore.

sions from each animal. Tissue, of course, plays a role in the interference pattern as well. The pattern, when dominated by multiple bubbles, would vary as the animal changed orientation and would be flat or nearly so when the axis of the array of inclusions is perpendicular or nearly so to the direction of incidence of the incoming signal.

While there were two to nine inclusions in any given siphonophore with a fragmented gas bubble, some were larger than others. After modeling the scattered signals, the best fits were obtained by assuming that two of the bubbles dominated the scattering (i.e., setting $N=2$ ). This approach was successful both modeling the single ping data (Fig. A3) and average echo data (Fig. A4). Since the orientation of the animal was not known for any given ping, the angle of orientation in the modeling of the single ping data was arbitrarily varied to move the position of the null and obtain a good fit to the data. Of course, for the average echo modeling, averages were calculated over all angles of orientation.

The average echo is shown to increase with decreasing frequency, an effect that is due to a combination of effects from both scattering by each individual bubble and the coherent addition of the scattering by the two bubbles within the animals at the lower frequencies (Fig. A4). Furthermore,
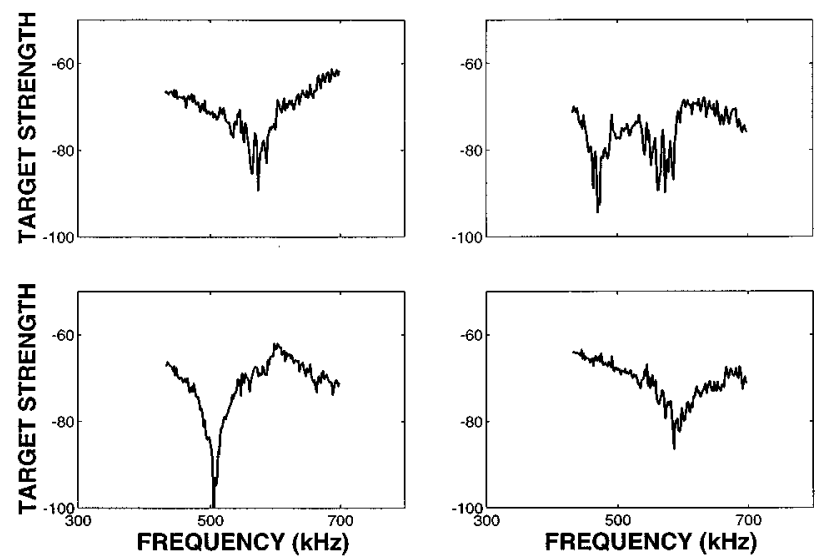

FIG. A2. Frequency spectrum (TS versus frequency) for four sequential echoes from a 24-mm-long siphonophore whose gas inclusion remained fragmented during the acoustics experiment. Throughout the experiment, it had two main bubbles each measuring about $1 \mathrm{~mm}$ in diameter with several smaller bubbles $0.5 \mathrm{~mm}$ in diameter and smaller. The pattern contained at least one null or dip for a significant fraction of the pings.

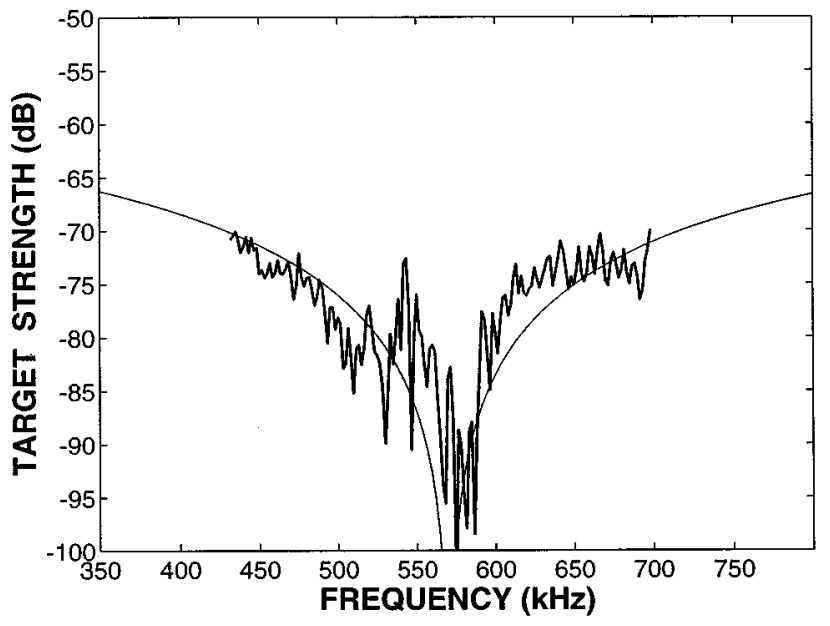

FIG. A3. Comparison between two-bubble model (thin curve) and single ping data (thick curve) from siphonophore whose gas inclusion remained fragmented throughout the experiment (animal described in Fig. A2 caption). Equation (A1) was used using Eq. (34) for $f_{1}$ and $N=2, a=0.7 \mathrm{~mm}$ (slightly above measured value, adjusted for best fit), $g=0.0012$, and $h$ $=0.22$. Here $D \sin \theta$ was adjusted arbitrarily to fit the location of the null (orientation is not known).

the deep nulls exhibited by those animals on a ping-by-ping basis were generally washed out during the averaging process, although some structure remained in this case.

To summarize, as a result of the net sampling process, the gas inclusions of some siphonophores apparently fragmented into multiple smaller inclusions (ranging from two to nine). The resultant scattering behavior of these animals was characterized by a very irregular pattern in single ping data, which is in sharp contrast to a much smoother one observed

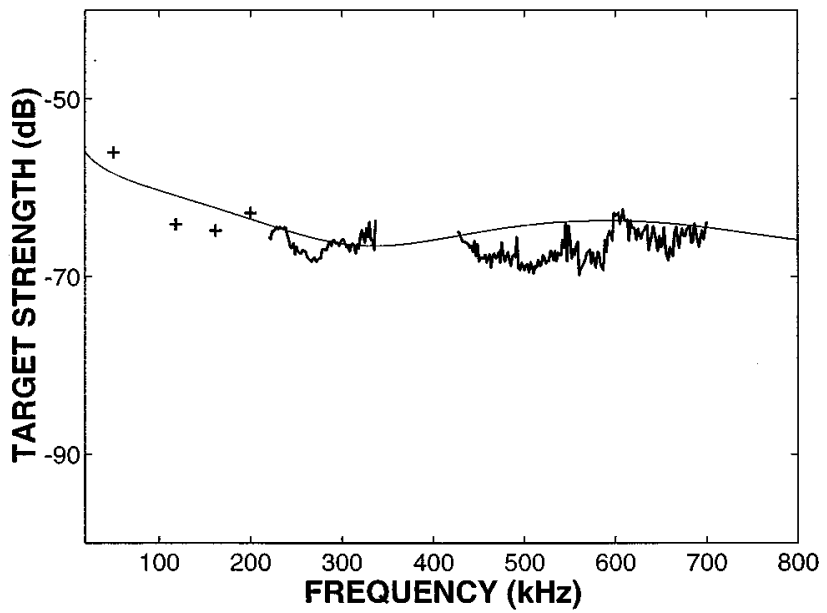

FIG. A4. Comparison between two-bubble model and 50-ping average of data from siphonophore whose gas inclusion remained fragmented throughout the experiment. Animal described in Fig. A2 caption. Equation (A2) used with the same (inclusion) radius of $0.7 \mathrm{~mm}$ used in this two-bubble simulation as in Fig. A3. In addition, a center-to-center separation, $D$, of $1.40 \mathrm{~mm}$ was required for a good fit. This should be compared with the 2.65 $\mathrm{mm}$ (before experiment) to $0.95 \mathrm{~mm}$ (after experiment) range of separations the animal experienced during the experiment. Thin curve is from predictions, thick irregular curve from broadband transducers, and " +" data from single frequency transducers. 
with some data with animals that contained only a single inclusion during the measurements. While the pattern is smoothed out once averaged over many pings, the resultant average level should be generally higher than that from a single gas inclusion of the same volume. For example, for a split of a single 1-mm-diam bubble into two bubbles, the increase in level averages about $1 \mathrm{~dB}$ at $200 \mathrm{kHz}$ over a range of separations.

Anderson, V. C. (1950). "Sound scattering from a fluid sphere," J. Acoust. Soc. Am. 22, 426-431.

Chu, D., and Stanton, T. K. (submitted). "Application of pulsecompression techniques to broadband acoustic scattering by live individual zooplankton,' submitted to J. Acoust. Soc. Am.

Chu, D., Foote, K. G., and Stanton, T. K. (1993). "Further analysis of target strength measurements of Antarctic krill at 38 and $120 \mathrm{kHz}$ : Comparison with deformed cylinder model and inference of orientation distribution,', J. Acoust. Soc. Am. 93, 2985-2988.

Chu, D., Stanton, T. K., and Wiebe, P. H. (1992). "'Frequency Dependence of Sound Backscattering from Live Individual Zooplankton,' ICES J. Mar. Sci. 49, 97-106.

Clay, C. S., and Heist, B. G. (1984). "Acoustic scattering by fish-acoustic models and a two parameter fit,'” J. Acoust. Soc. Am. 75, 1077-1083.

Felsen, L. B., and Lu, I. T. (1989). "Ray treatment of wave propagation on thin-walled curved elastic plates with truncations,' J. Acoust. Soc. Am. 86, 360-374

Foote, K. G. (1990). "Speed of sound in Euphausia superba,' J. Acoust. Soc. Am. 87, 1405-1408.

Foote, K. G., Everson, I., Watkins, J. L., and Bone, D. G. (1990). "Target strengths of Antarctic krill (Euphausia superba) at 38 and $120 \mathrm{kHz}$, , J. Acoust. Soc. Am. 87, 16-24.

Gaunaurd, G. C. (1985). "Sonar Cross Sections Partially Insonified by Finite Sound Beams,' IEEE J. Ocean Eng. OE-10(3), 213-230.

Goodman, R. R., and Stern, R. (1962). "Reflection and transmission of sound by elastic spherical shells,' J. Acoust. Soc. Am. 34, 338-344. For modal series coefficients involving spherical shells with an interior fluid different than that of the exterior fluid see, for example, Stanton (1990).

Ho, J. M. (1994). "Geometrical theory of acoustic scattering by thin elastic shells,', J. Acoust. Soc. Am. 96, 3115-3125.

Ho, J. M., and Felsen, L. B. (1990). "Nonconventional traveling wave formulations and ray-acoustic reductions for source-excited fluid-loaded thin elastic spherical shells,', J. Acoust. Soc. Am. 88, 2389-2414.

Jansson, P.-A. (1993). "Acoustic scattering from a rough sphere,' J. Acoust. Soc. Am. 93, 3032-3042.

Kaduchak, G. (1997). Personal communication.

Kaduchak, G., Kwiatkowski, C. S., and Marston, P. L. (1995). "Measurement and interpretation of the impulse response for backscattering by a thin spherical shell using a broad-bandwidth source that is nearly acoustically transparent,', J. Acoust. Soc. Am. 97, 2699-2708.

Kargl, S. G., and Marston, P. L. (1989). " Observations and modeling of the backscattering of short tone bursts from a spherical shell: Lamb wave echoes, glory, and axial reverberations,' J. Acoust. Soc. Am. 85, 10141028 .

Marston, P. L. (1992). "Geometrical and Catastrophe Optics Methods in Scattering,' in Physical Acoustics, edited by A. D. Pierce and R. N. Thurston (Academic, New York), Vol. 21.

Marston, P. L., Kargl, S. G., and Williams, K. L. (1990). "Rayleigh, Lamb, and Whispering Gallery Wave Contributions to Backscaterring from
Smooth Elastic Objects in Water Described by a Generalization of GTD," in Elastic Wave Propagation and Ultrasonic Nondestructive Evaluation, edited by S. K. Datta, J. D. Achenback, and Y. S. Rajapakse (Elsevier, Amsterdam), pp. 211-216.

Morse, P. M., and Ingard, K. U. (1968). Theoretical Acoustics (Princeton U. P., Princeton, NJ).

Norris, A. N., and Rebinsky, D. A. (1994). "Acoustic coupling to membrane waves on elastic shells," J. Acoust. Soc. Am. 95, 1809-1829.

Rebinsky, D. A., and Norris, A. N. (1995). "Benchmarking an acoustic coupling theory for elastic shells of arbitrary shape,' J. Acoust. Soc. Am. 98, 2368-2371.

Rice, S. O. (1954). "Mathematical Analysis of Random Noise,', in Selected Papers on Noise and Stochastic Processes, edited by N. Wax (Dover, New York), pp. 133-294.

Skudrzyk, E. (1971). The Foundation of Acoustics (Springer-Verlag, New York).

Stanton, T. K. (1984). "Sonar estimates of sea floor microroughness,' J. Acoust. Soc. Am. 75, 809-818.

Stanton, T. K. (1988). "Sound scattering by cylinders of finite length. I. Fluid cylinders,' J. Acoust. Soc. Am. 83, 55-63.

Stanton, T. K. (1989a). "Sound scattering by cylinders of finite length. III. Deformed cylinders,'” J. Acoust. Soc. Am. 86, 691-705.

Stanton, T. K. (1989b). "Simple approximate formulas for backscattering of sound by spherical and elongated objects,'” J. Acoust. Soc. Am. 86, 14991510 .

Stanton, T. K. (1990). "Sound scattering by spherical and elongated shelled bodies,' J. Acoust. Soc. Am. 88, 1619-1633.

Stanton, T. K. (1992). "Sound scattering by rough elongated elastic objects. I. Means of scattered field,' J. Acoust. Soc. Am. 92, 1641-1664.

Stanton, T. K., and Chu, D. (1992). "Sound scattering by rough elongated elastic objects. II: Fluctuations of scattered field,' J. Acoust. Soc. Am. 92, 1665-1678.

Stanton, T. K., Clay, C. S., and Chu, D. (1993a). "Ray representation of sound scattering by weakly scattering deformed fluid cylinders: Simple physics and application to zooplankton,', J. Acoust. Soc. Am. 94, 34543462 .

Stanton, T. K., Chu, D., Wiebe, P. H., and Clay, C. S. (1993b). “Average echoes from randomly oriented random-length finite cylinders: Zooplankton models," J. Acoust. Soc. Am. 94, 4363-3472.

Stanton, T. K., Chu, D., Wiebe, P. H., Martin, L. V., and Eastwood, R. L. (1998). "Sound scattering by several zooplankton groups. I. Experimental determination of dominant scattering mechanisms,', J. Acoust. Soc. Am. 103, 225-235.

Stanton, T. K., Wiebe, P. H., Chu, D., Benfield, M., Scanlon, L., Martin, L., and Eastwood, R. L. (1994a). "On Acoustic Estimates of Zooplankton Biomass,' ICES J. Mar. Sci. 51, 505-512.

Stanton, T. K., Wiebe, P. H., Chu, D., and Goodman, L. (1994b). “'Acoustic Characterization and Discrimination of Marine Zooplankton and Turbulence,', ICES J. Mar. Sci. 51, 469-479.

Wiebe, P. H., Greene, C. H., Stanton, T. K., and Burczynski, J. (1990). "Sound scattering by live zooplankton and micronekton: Empirical studies with a dual-beam acoustical system,' J. Acoust. Soc. Am. 88, 23462360

Yang, Y., Norris, A. N., and Couchman, L. S. (1995). “Acoustic scattering from fluid-loaded elastic shells: A Gaussian beam approach,' J. Acoust. Soc. Am. 98, 611-622.

Yang, Y., Norris, A. N., and Couchman, L. S. (1996). "Ray tracing over smooth elastic shells of arbitrary shape,' J. Acoust. Soc. Am. 99, 55-65. 